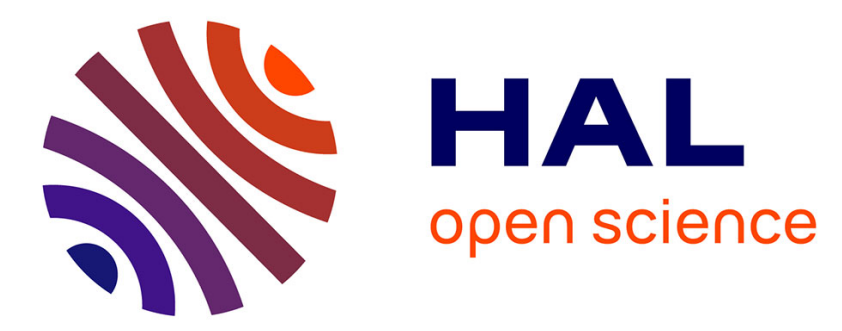

\title{
$\alpha-\beta$ Unsaturated Acylsilanes as Surrogates of Acrolein for Morita-Baylis-Hillman Reactions
}

\author{
Gangababu Marri, Frédéric Justaud, Saibal Das, René Grée
}

\section{To cite this version:}

Gangababu Marri, Frédéric Justaud, Saibal Das, René Grée. $\alpha-\beta$ Unsaturated Acylsilanes as Surrogates of Acrolein for Morita-Baylis-Hillman Reactions. European Journal of Organic Chemistry, 2019, 2019 (1), pp.56-65. 10.1002/ejoc.201801343 . hal-01944076

\section{HAL Id: hal-01944076 \\ https://hal-univ-rennes1.archives-ouvertes.fr/hal-01944076}

Submitted on 4 Dec 2018

HAL is a multi-disciplinary open access archive for the deposit and dissemination of scientific research documents, whether they are published or not. The documents may come from teaching and research institutions in France or abroad, or from public or private research centers.
L'archive ouverte pluridisciplinaire HAL, est destinée au dépôt et à la diffusion de documents scientifiques de niveau recherche, publiés ou non, émanant des établissements d'enseignement et de recherche français ou étrangers, des laboratoires publics ou privés. 


\title{
$\alpha-\beta$ Unsaturated Acylsilanes as Surrogates of Acrolein for Morita- Baylis-Hillman Reactions
}

\author{
Gangababu Marri, ${ }^{[a]}$ Frédéric Justaud, ${ }^{[b]}$ Saibal Das, ${ }^{*[a]}$ and René Grée*[b]
}

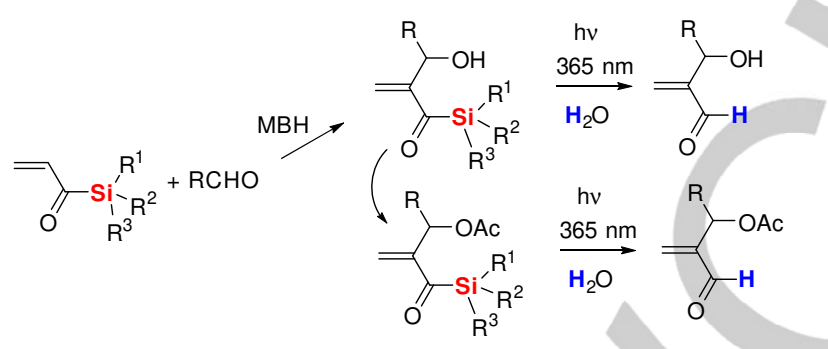

\begin{abstract}
: $\alpha-\beta$ unsaturated acylsilanes are excellent substrates for Morita-Baylis-Hillman (MBH) reactions, affording the expected adducts in good to excellent yields. In these derivatives, as well as the corresponding acetates, the acylsilanes can be smoothly transformed into aldehydes by irradiation at $365 \mathrm{~nm}$ in acetone or THF/water mixtures. Therefore $\alpha-\beta$ unsaturated acylsilanes are very useful surrogates for acrolein in $\mathrm{MBH}$ reactions, allowing easy preparation of simple and highly functionalized new building blocks for synthetic applications.
\end{abstract}

\section{Introduction}

The Morita-Baylis-Hillman ( $\mathrm{MBH}$ ) reaction has been established as a major $\mathrm{C}-\mathrm{C}$ bond forming reaction and the corresponding adducts proved to be particularly useful for the preparation of a very large number of molecules (Scheme 1, Eq. 1). ${ }^{[1]}$ Since this umpolung-type transformation involves the base-mediated condensation of an electrophilic alkene, such as an acrylate, with an aldehyde, the use of acrolein in this process is, at best, very difficult. In fact, only few papers reports such an application and important limitations have been usually observed. ${ }^{[2]}$ Therefore, in order to obtain the corresponding target molecules containing both an allylic alcohol and a vicinal aldehyde (Scheme 1, type- $\boldsymbol{A}$ and $-\boldsymbol{B})$, the classical process will be to transform another function, such as an ester group into the aldehyde by a reductionreoxidation process (Scheme 1, Eq. 2). ${ }^{[3]}$

On the other hand, the acylsilanes are well recognized as highly versatile functional groups with many applications in organic

[a] Dr. G. Marri, Dr. S. Das

CSIR-Indian Institute of Chemical Technology,

Department of Organic Synthesis and Process Chemistry,

Uppal Road, 500007 Hyderabad,

Telangana, India

E-Mail: saibal@iict.res.in

[b] Dr. F. Justaud, Dr. R. Grée

Univ Rennes, CNRS, ISCR (Institute for Chemical Sciences in

Rennes), UMR 6226, F-35000 Rennes, France.

E-mail: rene.gree@univ-rennes1.fr

http://blogperso.univ-rennes1/rene.gree/index.php/ synthesis. ${ }^{[4]}$ Since the acylsilane group can be transformed to aldehydes under very mild and neutral conditions (photolysis in water/organic solvents mixtures), ${ }^{[5-6]}$ we designed a strategy where $\alpha-\beta$ unsaturated acylsilanes could be employed in the MBH reaction and the adducts could be transformed directly and efficiently into the target molecules (Scheme 1, Eq. 3 and 4). The goal of this paper is to demonstrate, for the first time, that $\alpha-\beta$ unsaturated acylsilanes can be used in $\mathrm{MBH}$ reactions affording derivatives $\boldsymbol{C}$ and $\boldsymbol{D}$ and to validate this strategy towards type- $\boldsymbol{A}$ and $-\boldsymbol{B}$ functionalized enals which appear as versatile synthetic intermediates.

Morita-Baylis-Hillman reaction

$$
\text { Previous work }
$$

Scheme 1. Morita-Baylis-Hillman reaction and our working hypothesis toward the $A$ and $B$ target molecules

\section{Results and Discussion}

To the best of our knowledge, only one method has been reported to date for the preparation of molecules $\boldsymbol{A}$. It involves the condensation of the lithium anion of acrolein acetal with aldehydes, followed by deprotection of the acetal. (Scheme 2, Eq. 5). ${ }^{[7]}$ To be mentioned also, is the elegant method described 
recently which affords highly electrophilic 2-methylene-1,3dicarbonyl compounds $\boldsymbol{E}$ (Scheme 2, Eq. 6). ${ }^{[8]}$

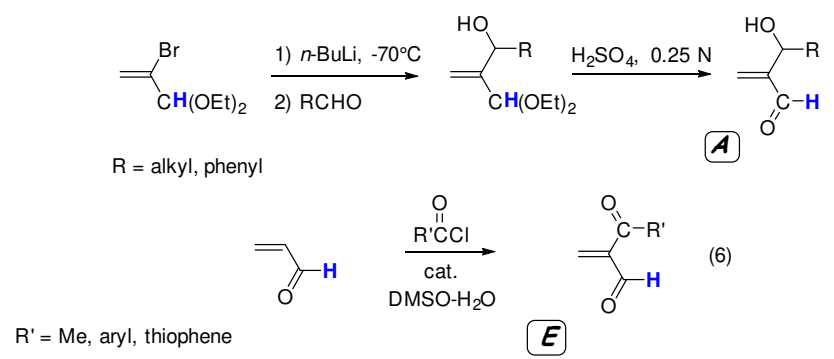

Scheme 2. Alternative methods reported for the synthesis of target molecules $A$ and alkenes $E$

The synthesis of the $\alpha-\beta$ unsaturated acylsilanes 3 has been performed by using slight optimizations (see Scheme 3 and experimental section) of literature procedures. Starting from allylic alcohol 1, and using retro-Brook reactions, the a-silyl allyl alcohols 2 have been obtained in good yields. The oxidation of alcohols 2 to the desired products $\mathbf{3}$ was performed efficiently by Swern method, or by using DMSO activated by $2,4,6$-Trichloro-1,3,5Triazine (TCT) ${ }^{[9]}$ Latter method proved to be more convenient on large scale reactions.

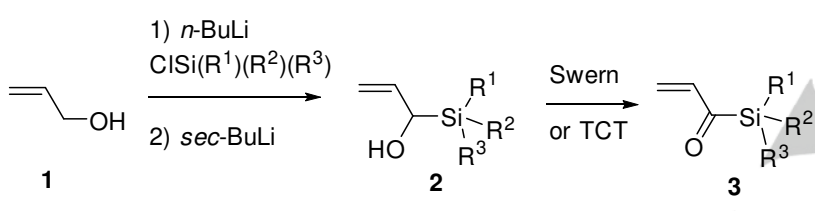

a: $R^{1}=R^{2}=R^{3}=E t ; b: R^{1}=R^{2}=M e, R^{3}=P h ; c: R^{1}=R^{2}=R^{3}=M e ;$

d: $R^{1}=R^{2}=R^{3}=\operatorname{iPr} ;$ e: $R^{1}=R^{2}=M e, R^{3}=t B u ; f: R^{1}=M e, R^{2}=R^{3}=P h$

Scheme 3. Synthesis of the $\alpha-\beta$ unsaturated acylsilanes

The $\alpha-\beta$ unsaturated acylsilane $\mathbf{3 a}$ and benzaldehyde were selected to explore the $\mathrm{MBH}$ reaction with a vinylic acylsilane (Scheme 4). ${ }^{[10]}$
$\underset{0}{\underset{O}{(} \mathrm{SiEt}_{3}}+\mathrm{RCHO} \underset{\mathrm{EtOAc,} \mathrm{RT}}{\mathrm{DABCO}}$

3a

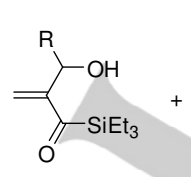

4a-12a

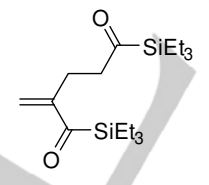

5
Scheme 4. Reactivity studies starting from acylsilane $\mathbf{3 a}$

After some experiments it was found that using DABCO as the nucleophilic catalyst and ethylacetate as the solvent, the desired adduct 4 a was obtained in $60 \%$ yield after six days at room temperature in the dark (Table 1, entry 1 ). As a byproduct the dimer 5a was also obtained, through a Rauhut-Currier (RC) type reaction. ${ }^{[11]}$ The $\mathrm{MBH}$ reaction was successfully extended to other aromatic aldehydes with nitro- and bromo-substituents (entries 25 ), as well as to the pyridine-3-carboxaldehyde (entry 6). Further, after two days reaction, the glyoxylate ethylester gave the $\mathrm{MBH}$ adduct only and in excellent yield (entry 7 ). The less reactive pentanal afforded the adduct 12a, but in lower yield and as a mixture with the dimer $5 a$ (entry 8 ). Clearly, the $\alpha-\beta$ unsaturated acylsilane $3 \mathbf{a}$ follows the general trends of the $\mathrm{MBH}$ reactions: the reactions are faster, and giving higher yields, with aldehydes bearing strong electron-withdrawing substituents. When the MBH reaction becomes slower and more difficult, the dimerization of the unsaturated starting material becomes competitive and affords higher yields of the Rauhut-Currier (RC) product.

(5) Table 1. MBH reaction studies with acylsilane $3 \mathrm{a}$ as model

\begin{tabular}{llllll}
\hline Entry & RCHO (equiv.) & $\begin{array}{l}\text { Time } \\
\text { (days) }\end{array}$ & $\begin{array}{l}\text { Ratio } \\
\text { (MBH:RC) }\end{array}$ & $\begin{array}{l}\text { Adduct } \\
(\%)\end{array}$ & $\mathbf{5 a ( \% )}$ \\
\hline 1 & PhCHO (1.5) & 6 & $72: 28$ & $4 a(60)$ & 16 \\
2 & 4-NO 2 PhCHO (1.2) & 2 & $100: 00$ & $\mathbf{6 a ~ ( 8 8 ) ~}$ & 0 \\
3 & 4- $\mathrm{BrPhCHO}(1.5)$ & 7 & $68: 32$ & $\mathbf{7 a ( 5 3 )}$ & 20 \\
4 & 2-BrPhCHO (1.5) & 6 & $80: 20$ & $\mathbf{8 a ~ ( 6 3 )}$ & 14 \\
5 & 3-BrPhCHO (1.5) & 6 & $96: 4$ & $\mathbf{9 a}(75)$ & 9 \\
6 & 3-PyrCHO (1.5) & 7 & $82: 18$ & $\mathbf{1 0 a}(68)$ & 13 \\
7 & $\mathrm{EtO}_{2} \mathrm{CCHO}(1.2)$ & 1.5 & $100: 00$ & $\mathbf{1 1 a}(93)$ & 0 \\
8 & $\mathrm{C}_{4} \mathrm{H}_{9} \mathrm{CHO}(2.0)$ & 10 & $50: 50$ & $\mathbf{1 2 a}(37)$ & 26 \\
\hline
\end{tabular}

[a] Ratios between products resulting from Morita-Baylis-Hillman adducts (MBH, 4a-12a) and Rauhut-Currier ( $\mathrm{RC}, 5 \mathrm{a})$ reaction, established by ${ }^{1} \mathrm{H}$ NMR analysis on the crude reaction mixtures.

In a next step, the $\mathrm{MBH}$ reaction was successfully extended to other acylsilanes (Scheme 5 and Table 2). On reaction with a few representative examples of aldehydes, this process could be developed with acylsilanes bearing five other commonly used groups: TMS, TIPS, TBDMS, $\mathrm{SiMe}_{2} \mathrm{Ph}$ and $\mathrm{SiPh}_{2} \mathrm{Me}$.
$\underset{O=}{\underset{3 b-3 f}{R_{i}^{3}}} \underset{R^{2}}{R^{1}}+R C H O \frac{\text { DABCO }}{\text { EtOAc, RT }}$

Scheme 5. Extension to other acylsilanes $\mathbf{3 b - 3 f}$

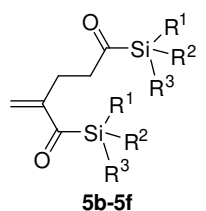

$5 b-5 f$
Table 2. MBH reactions with acylsilanes $\mathbf{3 a - 3 e}$

\begin{tabular}{|c|c|c|c|c|}
\hline Entry & $\mathrm{RCHO}$ (equiv.) & $\operatorname{Si}\left(R^{1}\right)\left(R^{2}\left(R^{3}\right)\right.$ & Adduct $(\%)^{\mathrm{a}}$ & $\begin{array}{l}5 a-5 f \\
(\%)\end{array}$ \\
\hline 1 & $\mathrm{PhCHO}(1.5)$ & $\mathrm{SiMe}_{2} \mathrm{Ph}$ & $4 b(40)$ & $5 \mathbf{b}(11)$ \\
\hline 2 & $4-\mathrm{NO}_{2} \mathrm{PhCHO}(1.1)$ & $\mathrm{SiMe}_{2} \mathrm{Ph}$ & $6 b(77)$ & 0 \\
\hline 3 & 4-BrPhCHO (1.2) & $\mathrm{SiMe}_{2} \mathrm{Ph}$ & $7 b(34)$ & $5 \mathbf{b}(20)$ \\
\hline 4 & $\mathrm{EtO}_{2} \mathrm{CCHO}(1.2)$ & $\mathrm{SiMe}_{2} \mathrm{Ph}$ & 11b (88) & 0 \\
\hline 5 & $4-\mathrm{NO}_{2} \mathrm{PhCHO}(1.2)$ & $\mathrm{Si}(\mathrm{Me})_{3}$ & $6 c(92)$ & 0 \\
\hline 6 & $\mathrm{EtO}_{2} \mathrm{CCHO}(1.5)$ & $\mathrm{Si}(\mathrm{Me})_{3}$ & 11c (22) & 0 \\
\hline 7 & $\mathrm{PhCHO}(1.5)$ & $\mathrm{Si}(i \mathrm{Pr})_{3}$ & $\mathbf{4 d}(10)$ & $5 \mathbf{d}(20)$ \\
\hline 8 & $4-\mathrm{NO}_{2} \mathrm{PhCHO}(1.2)$ & $\mathrm{Si}(\mathrm{PPr})_{3}$ & $6 d(30)$ & $5 \mathbf{d}(25)$ \\
\hline 9 & $\mathrm{EtO}_{2} \mathrm{CCHO}$ (1.5) & $\mathrm{Si}(\operatorname{PPr})_{3}$ & $11 d(70)$ & 0 \\
\hline 10 & $4-\mathrm{NO}_{2} \mathrm{PhCHO}(1.2)$ & $\mathrm{Si}-t \mathrm{BuMe}_{2}$ & $6 e(34)$ & 0 \\
\hline 11 & $\mathrm{EtO}_{2} \mathrm{CCHO}(1.5)$ & $\mathrm{Si}-\mathrm{BBM}_{2}$ & $11 \mathrm{e}(54)$ & 0 \\
\hline 12 & $4-\mathrm{NO}_{2} \mathrm{PhCHO}(1.2)$ & $\mathrm{SiPh}_{2} \mathrm{Me}$ & $6 f(72)$ & 0 \\
\hline 13 & $\mathrm{EtO}_{2} \mathrm{CCHO}$ (1.5) & $\mathrm{SiPh}_{2} \mathrm{Me}$ & $11 f(32)$ & 0 \\
\hline
\end{tabular}

[a] isolated yields of products resulting from Morita-Baylis-Hillman adducts (MBH, $\mathbf{4 b}-\mathbf{1 1 f})$ and Rauhut-Currier (RC, 5b-5d) reactions.

The results obtained show in general a similar tendency to those obtained with the TES group, and the MBH adducts $\mathbf{4 b - 1 2 f}$ were 
usually isolated in fair to good yields. However, in few cases (entries 6-8, 10 and 13), lower yields were obtained due to the lability of corresponding adducts.

The next step involved the protection of the allylic alcohol function. Based on our previous work in acylsilane chemistry, ${ }^{[5]}$ we selected the acetate group and the results are given in Scheme 6 and Table 3. The reactions gave the desired acetates 13a-15f in fair to good yields, although systematic optimization studies had to be performed and several conditions (A, B and C) were used depending upon the nature of the $R$ group and the silyl substituents (see Table 3 and experimental section).

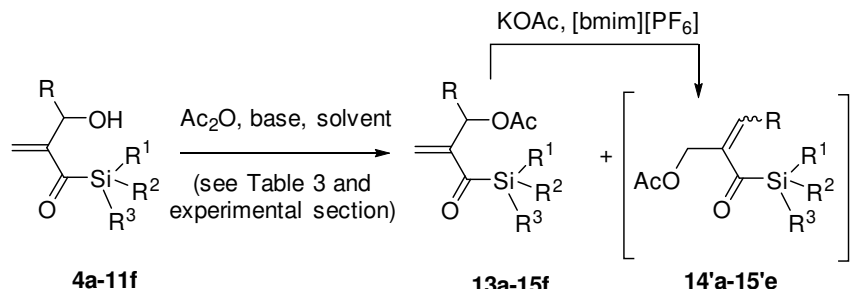

Scheme 6. Protection of acylsilanes MBH adducts

Table 3. Protection of $\mathrm{MBH}$ adducts

\begin{tabular}{|c|c|c|c|c|c|c|}
\hline Entry & $\mathrm{R}$ & $\begin{array}{l}\mathrm{Si}\left(\mathrm{R}^{1}\right) \\
\left(\mathrm{R}^{2}\left(\mathrm{R}^{3}\right)\right.\end{array}$ & $\begin{array}{l}\text { Cond. } \\
\text { [a] }\end{array}$ & $\begin{array}{c}\text { Time } \\
(\mathrm{h})\end{array}$ & Adduct & $\begin{array}{l}\text { Yield } \\
(\%)\end{array}$ \\
\hline 1 & $\mathrm{Ph}$ & $\mathrm{SiEt}_{3}$ & $A$ & 20 & $13 a$ & 63 \\
\hline 2 & $4-\mathrm{NO}_{2} \mathrm{Ph}$ & $\mathrm{SiEt}_{3}$ & $A$ & 6 & $14 a$ & 92 \\
\hline 3 & $4-\mathrm{NO}_{2} \mathrm{Ph}$ & $\mathrm{SiEt}_{3}$ & $A$ & 48 & $\begin{array}{l}14 a \\
+(14 ' a)\end{array}$ & $81(18)$ \\
\hline 4 & $4-\mathrm{NO}_{2} \mathrm{Ph}$ & $\mathrm{SiEt}_{3}$ & $\mathrm{C}$ & 6 & $14 a$ & 82 \\
\hline 5 & $4-\mathrm{NO}_{2} \mathrm{Ph}$ & $\mathrm{SiEt}_{3}$ & $D$ & 2 & 14 'a & 64 \\
\hline 6 & $\mathrm{EtO}_{2} \mathrm{C}$ & $\mathrm{SiEt}_{3}$ & $A$ & 40 & $15 a$ & 88 \\
\hline 7 & $\mathrm{Ph}$ & $\mathrm{SiPhMe}_{2}$ & $B$ & 6 & $13 b$ & 76 \\
\hline 8 & $4-\mathrm{NO}_{2} \mathrm{Ph}$ & $\mathrm{SiPhMe}_{2}$ & B & 5 & $14 b$ & 80 \\
\hline 9 & $\mathrm{EtO}_{2} \mathrm{C}$ & $\mathrm{SiPhMe}_{2}$ & B & 5 & $15 b$ & 67 \\
\hline 10 & $4-\mathrm{NO}_{2} \mathrm{Ph}$ & $\mathrm{SiMe}_{3}$ & A & 0.5 & $14 c$ & 72 \\
\hline 11 & $4-\mathrm{NO}_{2} \mathrm{Ph}$ & $\mathrm{SiMe}_{3}$ & $D$ & 2 & 14 'c & 72 \\
\hline 12 & $\mathrm{EtO}_{2} \mathrm{C}$ & $\mathrm{SiMe}_{3}$ & A & 5 & $15 c$ & 67 \\
\hline 13 & $\mathrm{EtO}_{2} \mathrm{C}$ & $\mathrm{SiMe}_{3}$ & C & 24 & $15 c$ & 75 \\
\hline 14 & $4-\mathrm{NO}_{2} \mathrm{Ph}$ & $\mathrm{Si}^{i} \mathrm{Pr}_{3}$ & $B$ & 5 & $14 d$ & 51 \\
\hline 15 & $\mathrm{EtO}_{2} \mathrm{C}$ & $\mathrm{Si}^{\mathrm{i} P r_{3}}$ & B & 3 & $15 d$ & 70 \\
\hline 16 & $4-\mathrm{NO}_{2} \mathrm{Ph}$ & $\mathrm{Si}^{t} \mathrm{BuMe}_{2}$ & C & 48 & $14 \mathrm{e}$ & 68 \\
\hline 17 & $4-\mathrm{NO}_{2} \mathrm{Ph}$ & $\mathrm{Si}^{t} \mathrm{BuMe}_{2}$ & D & 2 & 14 'e & 81 \\
\hline 18 & $\mathrm{EtO}_{2} \mathrm{C}$ & $\mathrm{Sit}^{\mathrm{t} B u M e}{ }_{2}$ & C & 24 & $15 e$ & 84 \\
\hline 19 & $\mathrm{EtO}_{2} \mathrm{C}$ & $\mathrm{Si}^{t} \mathrm{BuMe}{ }_{2}$ & D & 2 & 15 'e & 64 \\
\hline 20 & $4-\mathrm{NO}_{2} \mathrm{Ph}$ & $\mathrm{SiPh}_{2} \mathrm{Me}$ & $A$ & 0.2 & $14 f$ & $79[b]$ \\
\hline 21 & $4-\mathrm{NO}_{2} \mathrm{Ph}$ & $\mathrm{SiPh}_{2} \mathrm{Me}$ & $\mathrm{C}$ & 48 & $14 f$ & 54 \\
\hline 22 & $\mathrm{EtO}_{2} \mathrm{C}$ & $\mathrm{SiPh}_{2} \mathrm{Me}$ & C & 48 & $15 f$ & 77 \\
\hline
\end{tabular}

[a] Conditions $A$ : $\mathrm{Ac}_{2} \mathrm{O}$ (2 equiv), $\mathrm{Et}_{3} \mathrm{~N}$ ( 3 equiv), DMAP ( 0.1 equiv) $\mathrm{DCM}, 0{ }^{\circ} \mathrm{C}$ to rt.; $\mathrm{B}: \mathrm{Ac}_{2} \mathrm{O}$ (2 equiv), DMAP ( 0.1 equiv), DCM, rt; $\mathrm{C}: \mathrm{Ac}_{2} \mathrm{O}$ (5 equiv), $\mathrm{NaHCO}_{3}$ (2 equiv), DCM, rt ; D: KOAc (1.5 equiv), [bmim]PF $6,50{ }^{\circ} \mathrm{C}, 2 \mathrm{~h}$.

[b] Temperature of the reaction: $-10^{\circ} \mathrm{C}$.

The main reason was that, in several cases, during this protection step, the products resulting from an allylic transposition 14'-15' were also obtained. For instance, acetates 13a and 14a were easily obtained from $\mathbf{4 a}$ and $\mathbf{6} \mathbf{a}$ using conditions $\mathrm{A}$ (Table 3 , entries 1 and 2). However, increasing the reaction time afforded some transposed allylic derivative 14 'a (18\% of $E$ isomer) (entry 3). Therefore, we had to use, in parallel, a method to isolate the transposed products in order to have authentic samples of such molecules. Reaction of allylic acetate $14 a$ with potassium acetate in ionic liquids (conditions D), ${ }^{[12]}$ afforded efficiently the transposition product 14'a (entry $5, E$ isomer only). The stereochemistry of the $E$ isomer of 14 'a was established by NoE experiments. By using these methods, all desired derivatives 1315 could be prepared under appropriate conditions (Table 3), while the regioisomeric derivatives $1 \mathbf{1 4}^{\prime}, \mathbf{1 5}^{\prime}$ ' were obtained as well by using conditions $\mathrm{D}$.

The, final, key step involved the transformation of the acylsilane units into aldehydes (Scheme 7). Based on our recent work, we have employed photolytic conditions in a (1:2) water: acetone mixture and classical UV lamps at $365 \mathrm{~nm} \cdot{ }^{[13]}$ By using these very simple conditions the target molecules 16-18, with three representative $\mathrm{R}$ groups $\left(\mathrm{Ph}, 4-\mathrm{NO}_{2} \mathrm{Ph}\right.$ and $\mathrm{EtO}_{2} \mathrm{C}$ ) were obtained in excellent yields from derivatives 13a-15f (Table 4). Importantly this method proved to be successful, independently of the nature of the silicon protecting groups (TES, TMS, TIPS and $\mathrm{SiPhMe}_{2}$ ) and the enals 16-18 were obtained in good to excellent yields (69$95 \%$, Table 4, entries 1-13).

$$
\text { Acetone/ } \mathrm{H}_{2}
$$

Scheme 7. Conversion of acylsilanes $\mathrm{MBH}$ and $\mathrm{RC}$ adducts into enals

Further, it proved to be even possible to perform this transformation directly on the first, non-protected, $\mathrm{MBH}$ adducts 4a-11f. In that case we changed the organic co-solvent from acetone to THF, for solubility reasons. These reactions afforded in fair to good yields the target molecules 19-21, with the same three $\mathrm{R}$ groups as before (Table 4, entries 14-22).

Finally, to complete this study we considered the possibility to use the same transformation of acylsilanes into aldehydes on the Rauhut-Currier adducts $\mathbf{5 a}$ to $\mathbf{5 d}$ (Scheme 7). The reaction 
afforded cleanly, and in good to excellent yields, the desired compounds 22 - 24 with both an enal and a remote acylsilane unit.

Table 4. Transformation of acylsilanes MBH adducts into enals.

\begin{tabular}{|c|c|c|c|c|c|}
\hline Entry & $\begin{array}{l}\text { Starting } \\
\text { Material }\end{array}$ & Product & $\mathrm{R}$ & $\begin{array}{l}\text { Time } \\
\text { (h) }\end{array}$ & $\begin{array}{l}\text { Yield } \\
(\%)\end{array}$ \\
\hline 1 & $13 a$ & 16 & $\mathrm{Ph}$ & 48 & 95 \\
\hline 2 & $14 a$ & 17 & $4-\mathrm{NO}_{2} \mathrm{Ph}$ & 48 & 92 \\
\hline 3 & $15 a$ & 18 & $\mathrm{EtO}_{2} \mathrm{C}$ & 40 & 53 \\
\hline 4 & $13 b$ & 16 & $\mathrm{Ph}$ & 24 & 88 \\
\hline 5 & $14 b$ & 17 & $4-\mathrm{NO}_{2} \mathrm{Ph}$ & 40 & 88 \\
\hline 6 & $15 b$ & 18 & $\mathrm{EtO}_{2} \mathrm{C}$ & 20 & 83 \\
\hline 7 & $15 c$ & 18 & $\mathrm{EtO}_{2} \mathrm{C}$ & 40 & 95 \\
\hline 8 & $14 d$ & 17 & $4-\mathrm{NO}_{2} \mathrm{Ph}$ & 20 & 75 \\
\hline 9 & $15 d$ & 18 & $\mathrm{EtO}_{2} \mathrm{C}$ & 6 & 80 \\
\hline 10 & $14 e$ & 17 & $4-\mathrm{NO}_{2} \mathrm{Ph}$ & 20 & 91 \\
\hline 11 & $15 e$ & 18 & $\mathrm{EtO}_{2} \mathrm{C}$ & 24 & 95 \\
\hline 12 & $14 f$ & 17 & $4-\mathrm{NO}_{2} \mathrm{Ph}$ & 20 & 69 \\
\hline 13 & $15 f$ & 18 & $\mathrm{EtO}_{2} \mathrm{C}$ & 24 & 81 \\
\hline 14 & $4 a$ & 19 & $\mathrm{Ph}$ & 20 & 61 \\
\hline 15 & $6 a$ & 20 & $4-\mathrm{NO}_{2} \mathrm{Ph}$ & 48 & 78 \\
\hline 16 & $11 a$ & 21 & $\mathrm{EtO}_{2} \mathrm{C}$ & 48 & 81 \\
\hline 17 & $6 c$ & 20 & $4-\mathrm{NO}_{2} \mathrm{Ph}$ & 48 & 86 \\
\hline 18 & $11 \mathrm{c}$ & 21 & $\mathrm{EtO}_{2} \mathrm{C}$ & 48 & 53 \\
\hline 19 & $6 e$ & 20 & $4-\mathrm{NO}_{2} \mathrm{Ph}$ & 48 & 86 \\
\hline 20 & $11 \mathrm{e}$ & 21 & $\mathrm{EtO}_{2} \mathrm{C}$ & 48 & 63 \\
\hline 21 & $6 f$ & 20 & $4-\mathrm{NO}_{2} \mathrm{Ph}$ & 48 & 93 \\
\hline 22 & $11 f$ & 21 & $\mathrm{EtO}_{2} \mathrm{C}$ & 48 & 74 \\
\hline 23 & $5 a$ & 22 & - & 24 & 60 \\
\hline 24 & $5 b$ & 23 & - & 40 & 90 \\
\hline 25 & $5 d$ & 24 & - & 8 & 90 \\
\hline
\end{tabular}

This result is in agreement with literature data indicating that conjugated acylsilanes are more easily cleaved under photolytic conditions that non conjugated substrates. ${ }^{[6]}$ Similar reactions have been performed starting from the transposed $(E)$ acylsilane 14'a. However, they did not afford the expected conjugated aldehyde, and they gave only $E-Z$ equilibration of this molecule, without hydrolysis of the acylsilane unit.

\section{Conclusions}

In summary, we have disclosed a short and efficient approach toward new acrolein-type derivatives, functionalized in position 2. Taking into account their multiple reactivity profile, these molecules appear as versatile building blocks ${ }^{[14]}$ for the preparation of natural products, as well as various type of bioactive compounds.

\section{Experimental section}

General Information: All anhydrous reactions were performed in oven-dried round-bottomed flasks under a dry argon atmosphere. Air and moisture-sensitive compounds were introduced via syringes or cannulae, using standard inert atmosphere techniques. Reactions were monitored by thin layer chromatography (TLC) using E. Merck silica gel plates and components were visualized by illumination with shortwavelength UV light and/or staining ( $p$-anisaldehyde or basic $\mathrm{KMnO}_{4}$ ). All reagents were used as they were received from commercial suppliers, unless otherwise noted. THF was dried in the presence of sodium metal using benzophenone as indicator and distilled prior to use. Anhydrous $\mathrm{CH}_{2} \mathrm{Cl}_{2}$ was prepared by refluxing in the presence of $\mathrm{CaH}_{2}$ and distilled right before use. We used a Vilber Lourmat VL-6.LC lamp equiped with two 6W bulbs at $365 \mathrm{~nm}$ for the small scale reactions, or a LT5W T8/010 UV Dudexa lamp from Narva for the larger scale reactions. ${ }^{1} \mathrm{H}$ NMR spectra were recorded at 300,400 and $500 \mathrm{MHz}$, and ${ }^{13} \mathrm{C}$ NMR spectra were recorded at 75,101 and $126 \mathrm{MHz}$, respectively in $\mathrm{CDCl}_{3}$. The residual peak of $\mathrm{CHCl}_{3}$ was set at 7.26 ppm for ${ }^{1} \mathrm{H}$ $\mathrm{NMR}$ and the central peak of $\mathrm{CDCl}_{3}$ was set at $77.0 \mathrm{ppm}$ for ${ }^{13} \mathrm{C}$ NMR. All products were purified by flash column chromatography on silica gel (100 - 200 mesh).

General experimental procedure for the preparation of $\alpha$ hydroxyallylsilanes (2): to a solution of allyl alcohol 1 (1 equiv) in anhydrous THF was added $n$-BuLi (1.2 equiv) at $-78^{\circ} \mathrm{C}$. After 30 min stirring at the same temperature, $\mathrm{SiR}^{1} \mathrm{R}^{2} \mathrm{R}^{3} \mathrm{Cl}$ (1 equiv) in dry THF was added in dropwise. Then, the reaction was warmed to room temperature and stirred for $18 \mathrm{~h}$. Next, sec-BuLi (1.05 equiv) was added in dropwise at $-78^{\circ} \mathrm{C}$. After stirring for $2 \mathrm{~h}$ at $50{ }^{\circ} \mathrm{C}$, the reaction mixture was quenched with aqueous $\mathrm{NH}_{4} \mathrm{Cl}$ at $-78{ }^{\circ} \mathrm{C}$, and then diluted with $\mathrm{Et}_{2} \mathrm{O}$. The organic phase was collected and the aqueous phase was extracted with $\mathrm{Et}_{2} \mathrm{O}$ for twice. Combined organic phases were dried with $\mathrm{Na}_{2} \mathrm{SO}_{4}$, concentrated under reduce pressure. The residue was purified by column chromatography on silica gel to afford (2) as a colourless oil. With this procedure we prepared all $\alpha$-hydroxyallylsilanes (2a to $2 \mathrm{f})$.

General experimental procedure for preparation of acryloylsilanes by Swern protocol (3a-3f): to a solution of trifluoroacetic anhydride (1.5 equiv) in $\mathrm{CH}_{2} \mathrm{Cl}_{2}$ was added DMSO ( 2 equiv) dropwise at $-78{ }^{\circ} \mathrm{C}$. After $30 \mathrm{~min}$ stirring at the same temperature, $\alpha$ - hydroxyallylsilanes (2a-2f) (1 equiv) in $\mathrm{CH}_{2} \mathrm{Cl}_{2}$ were added in dropwise. Then the reaction was stirred for $1 \mathrm{~h}$ at $78^{\circ} \mathrm{C}$, and followed by adding TEA ( 3 equiv) slowly. After stirring for another $1 \mathrm{~h}$ at $-78^{\circ} \mathrm{C}$, the reaction mixture was quenched with water and warmed to room temperature. The organic phase was collected and the aqueous phase was extracted twice with $\mathrm{CH}_{2} \mathrm{Cl}_{2}$. The organic phases were combined, dried with $\mathrm{MgSO}_{4}$ and concentrated in vacuum. The residue was purified by column chromatography on silica gel to afford acryloylsilanes (3a-3f) as bright yellow liquids.

General experimental procedure for preparation of acryloylsilanes by using TCT (3a-3f): DMSO (5 equiv) was added slowly to a solution of 2,4,6-Trichloro-1,3,5-Triazine (TCT,1.2 equiv) in THF, stirred and maintained at $-30{ }^{\circ} \mathrm{C}$ for 30 min. Then, a solution of $\alpha$-hydroxyallylsilane 3 (1.0 equiv) in dry THF was added dropwise and stirring continued for a further 30 min. Finally $\mathrm{Et}_{3} \mathrm{~N}$ (4 equiv) was added with an additional 30 min of stirring. The mixture was poored in pentane under vigourous stirring with appearance of a sticky mass of TEA salt, which was discarded. The rest of the cloudy solution was stirred with an excess of $\mathrm{MgSO}_{4}$ while addition of a small amount of water $(0.5$ to $1.0 \mathrm{ml}$ ) permitted the clearance of this solution. Then, it was filtrated and the volatiles were evaporated at $450 \mathrm{Mbar}$ with a water bath at $45^{\circ} \mathrm{C}$. Additional pentane was added twice to ensure the total impoverishment of the solvent by azeotropic distillation. 
The product was purified by column chromatography on silica gel to afford acryloylsilanes (3a-3f) as bright yellow liquids.

1-(Triethylsilyl)prop-2-en-1-one (3a): $\mathrm{R}_{f}$ : 0.8 (5:95 EtOAc/nHexane); Yield 88\%, as a yellow oil; FT-IR (film) 2954, 2876, 1598 $1180,1002,730 \mathrm{~cm}^{-1} ;{ }^{1} \mathrm{H}$ NMR $\left(500 \mathrm{MHz}, \mathrm{CDCl}_{3}\right) \delta=6.44(\mathrm{dd}, J$ $=17.9,10.8 \mathrm{~Hz}, 1 \mathrm{H}), 6.03(\mathrm{dd}, J=17.9,1.1 \mathrm{~Hz} 1 \mathrm{H}), 5.83(\mathrm{dd}, J=$ $10.8,1.1 \mathrm{~Hz}, 1 \mathrm{H}), 0.93(\mathrm{t}, J=7.8 \mathrm{~Hz}, 9 \mathrm{H}), 0.75(2 \times \mathrm{q}, J=7.8,1.0$ $\mathrm{Hz}, 6 \mathrm{H}) \mathrm{ppm} ;{ }^{13} \mathrm{C} \mathrm{NMR}\left(126 \mathrm{MHz}, \mathrm{CDCl}_{3}\right) \delta=237.7,142.1,127.2$, 7.1, $2.9 \mathrm{ppm}$; HRMS (ESI +ve) Exact mass calculated for $\mathrm{C}_{9} \mathrm{H}_{18} \mathrm{ONaSi}[\mathrm{M}+\mathrm{Na}]^{+}:$193.1019, found: 193.1022.

1-(Dimethyl(phenyl)silyl)prop-2-en-1-one (3b): $\mathrm{R}_{f}: 0.6$ (5:95 EtOAc/n-Hexane); Yield $80 \%$ as a yellow oil; FT-IR (film) 2960, $2871,1600,1428,1250,1183,1110,1024,955,815,783,697$, $653 \mathrm{~cm}^{-1} ;{ }^{1} \mathrm{H} \mathrm{NMR}\left(500 \mathrm{MHz}, \mathrm{CDCl}_{3}\right) \delta=7.56-7.51(\mathrm{~m}, 2 \mathrm{H}), 7.43-$ $7.34(\mathrm{~m}, 3 \mathrm{H}), 6.41$ (dd, $J=17.9,10.8 \mathrm{~Hz}, 1 \mathrm{H}), 5.99(\mathrm{dd}, J=17.9$, $0.9 \mathrm{~Hz}, 1 \mathrm{H}), 5.86$ (dd, $J=10.8,0.9 \mathrm{~Hz}, 1 \mathrm{H}), 0.53(\mathrm{~s}, 6 \mathrm{H}) \mathrm{ppm} ;{ }^{13} \mathrm{C}$ NMR $\left(75 \mathrm{MHz}, \mathrm{CDCl}_{3}\right) \delta=235.8,141.2,135.1,133.9,129.9$, 129.4, 128.2, -3.6 ppm; HRMS (ESI +ve) Exact mass calculated for $\mathrm{C}_{11} \mathrm{H}_{15} \mathrm{OSi}[\mathrm{M}+\mathrm{H}]^{+} ;$191.0892, found: 191.0890.

1-(Trimethylsilyl)prop-2-en-1-one (3c): $\mathrm{R}_{f}: 0.7$ (5:95 EtOAc/nHexane); Yield $91 \%$ as a yellow oil; FT-IR (film) 2960, 1734, 1460 , 1259, 1083, 842, $800 \mathrm{~cm}^{-1} ;{ }^{1} \mathrm{H}$ NMR $\left(300 \mathrm{MHz}, \mathrm{CDCl}_{3}\right) \delta 6.43$ (dd, $J=18.0,10.7 \mathrm{~Hz}, 1 \mathrm{H}), 6.12(\mathrm{dd}, J=18.0,1.0 \mathrm{~Hz}, 1 \mathrm{H}), 5.97(\mathrm{dd}$, $J=10.7,1.0 \mathrm{~Hz}, 1 \mathrm{H}), 0.27(\mathrm{~s}, 9 \mathrm{H}) \mathrm{ppm} ;{ }^{13} \mathrm{C}$ NMR $(75 \mathrm{MHz}$, $\left.\mathrm{CDCl}_{3}\right) \delta 237.8,141.4,128.5,-2.3$ ppm; HRMS (ESI +ve) Exact mass calculated for $\mathrm{C}_{6} \mathrm{H}_{13} \mathrm{OSi}[\mathrm{M}+\mathrm{H}]^{+} ; 129.0730$, found: 129.0731

1-(Triisopropylsilyl)prop-2-en-1-one $\quad$ (3d): $\quad R_{f}: \quad 0.8 \quad(5: 95$ EtOAc/n-Hexane); Yield 55\% as a yellow oil; FT-IR (film) 2944, $2867,1637,1576,1464,1390,1177,986,882,677,642 \mathrm{~cm}^{-1} ;{ }^{1} \mathrm{H}$ $\operatorname{NMR}\left(400 \mathrm{MHz}, \mathrm{CDCl}_{3}\right) \delta=6.70(\mathrm{dd}, J=17.4,10.6 \mathrm{~Hz}, 1 \mathrm{H}), 5.96$ (dd, $J=17.4,1.5 \mathrm{~Hz}, 1 \mathrm{H}$ ), 5.56 (dd, $J=10.6,1.5 \mathrm{~Hz}, 1 \mathrm{H}$ ), 1.34 (hept, $J=7.5 \mathrm{~Hz}, 3 \mathrm{H}), 1.11$ (d, $J=7.5 \mathrm{~Hz}, 18 \mathrm{H}$ ) ppm; ${ }^{13} \mathrm{C}$ NMR $\left(101 \mathrm{MHz}, \mathrm{CDCl}_{3}\right) \delta=236.7,141.0,123.6,18.5,11.1 \mathrm{ppm}$; HRMS: (ESI -ve) Exact mass calculated for $\mathrm{C}_{12} \mathrm{H}_{25} \mathrm{OSi}[\mathrm{M}+\mathrm{H}]^{+}$; 213.1675, found: 213.1678 .

1-(Tert-butyldimethylsilyl)prop-2-en-1-one (3e): $\mathrm{R}_{f}: 0.75$ (5:95 EtOAc/n-Hexane); Yield: $89 \%$ as a yellow oil; FT-IR (film) 2854, 1644, 1463, 1259, 1082, 1018, $800 \mathrm{~cm}^{-1} ;{ }^{1} \mathrm{H}$ NMR $(300 \mathrm{MHz}$, $\left.\mathrm{CDCl}_{3}\right) \delta=6.59(\mathrm{dd}, J=17.7,10.7 \mathrm{~Hz}, 1 \mathrm{H}), 6.04(\mathrm{dd}, J=17.7$, $1.3 \mathrm{~Hz}, 1 \mathrm{H}$ ), 5.77 (dd, $J=10.7,1.3 \mathrm{~Hz}, 1 \mathrm{H}$ ), 0.95 (s, 9H), 0.25 (s, $6 \mathrm{H}) \mathrm{ppm} ;{ }^{13} \mathrm{C} \mathrm{NMR}\left(75 \mathrm{MHz}, \mathrm{CDCl}_{3}\right) \delta=236.9,141.4,126.4$, 26.5, 16.6, -6.2 ppm; HRMS (ESI -ve) Exact mass calculated for $\mathrm{C}_{9} \mathrm{H}_{19} \mathrm{OSi}[\mathrm{M}+\mathrm{H}]^{+} ;$171.1199, found: 171.1202 .

1-(Methyldiphenylsilyl)prop-2-en-1-one (3f): $R_{f}: 0.6$ (5:95 EtOAc/n-Hexane); Yield $73 \%$ as a yellow oil; FT-IR (film) 3048 , $1638,1486,1427,1251,1185,1110,997,790,727,696 \mathrm{~cm}^{-1} ;{ }^{1} \mathrm{H}$ $\operatorname{NMR}\left(300 \mathrm{MHz}, \mathrm{CDCl}_{3}\right) \delta=7.64-7.58(\mathrm{~m}, 4 \mathrm{H}), 7.50-7.35(\mathrm{~m}, 6 \mathrm{H})$, $6.57(\mathrm{dd}, J=17.8,10.8 \mathrm{~Hz}, 1 \mathrm{H}), 6.02(\mathrm{dd}, J=17.8,0.9 \mathrm{~Hz}, 1 \mathrm{H})$, 5.85 (dd, $J=10.8,0.9 \mathrm{~Hz}, 1 \mathrm{H}), 0.81(\mathrm{~s}, 3 \mathrm{H}) \mathrm{ppm} ;{ }^{13} \mathrm{C}$ NMR $(75$ $\left.\mathrm{MHz}, \mathrm{CDCl}_{3}\right) \delta=233.7,141.4,135.1,133.2,130.1,129.6,128.2$, -4.3 ppm; HRMS (ESI +ve) Exact mass calculated for $\mathrm{C}_{16} \mathrm{H}_{16} \mathrm{ONaSi}[\mathrm{M}+\mathrm{Na}]^{+} ; 275.0862$, found: 275.0864 .
General experimental procedure for the preparation of $\alpha$ functional $\alpha, \beta$-unsaturated acylsilanes via $\mathrm{MBH}$ reactions:

To a solution of acryloylsilane 3 in EtOAc was added the aldehyde, followed by $\mathrm{DABCO}$ at $0^{\circ} \mathrm{C}$ and reached room temperature with stirring. After consumption of the starting material, water was added and the organic phase was separated and washed with water. Aqueous phases were extracted with EtOAc twice and the organic phases gathered, dried with $\mathrm{MgSO}_{4}$ and concentrated under reduced pressure. The crude product was purified by column chromatography.

2-(Hydroxy(phenyl)methyl)-1-(triethylsilyl)prop-2-en-1-one (4a): yield $60 \%$ as a yellow oil. FT-IR (film) 3447, 2954, 2875, 1594, 1454, 1019, 734, $697 \mathrm{~cm}^{-1} ;{ }^{1} \mathrm{H}$ NMR $\left(500 \mathrm{MHz}, \mathrm{CDCl}_{3}\right) \delta=$ $7.32-7.25(\mathrm{~m}, 4 \mathrm{H}), 7.23-7.19(\mathrm{~m}, 1 \mathrm{H}), 6.14(\mathrm{~d}, J=1.3 \mathrm{~Hz}, 1 \mathrm{H})$, 6.09 (s, $1 \mathrm{H}), 5.53(\mathrm{~d}, J=4.6 \mathrm{~Hz}, 1 \mathrm{H}), 3.51(\mathrm{~d}, J=4.9 \mathrm{~Hz}, 1 \mathrm{H})$, 0.90 (t, $J=7.8 \mathrm{~Hz}, 9 \mathrm{H}), 0.75-0.77(2 \times \mathrm{q}, J=7.8 \mathrm{~Hz}, 6 \mathrm{H}) \mathrm{ppm} ;{ }^{13} \mathrm{C}$ NMR $\left(126 \mathrm{MHz}, \mathrm{CDCl}_{3}\right) \delta 239.3,155.7,141.9,128.2,127.9$, 127.4, 126.6, 72.2, 7.2, 3.5 ppm; HRMS (ESI +ve) Exact mass calculated for $\mathrm{C}_{16} \mathrm{H}_{24} \mathrm{O}_{2} \mathrm{NaSi}[\mathrm{M}+\mathrm{Na}]^{+}:$299.1437, found: 299.1438 .

2-methylene-1,5-bis(triethylsilyl)pentane-1,5-dione (5a): yield $16 \%$ as a yellow oil. FT-IR (film) 2954, 2876, 1641, 1595, 1414, $1004,719 \mathrm{~cm}^{-1} ;{ }^{1} \mathrm{H} \mathrm{NMR}\left(300 \mathrm{MHz}, \mathrm{CDCl}_{3}\right) \delta=6.04($ broad s, $1 \mathrm{H})$, 5.95 (s, 1H), 2.68-2.60 (t, $J=7.4 \mathrm{~Hz}, 2 \mathrm{H}$ ), 2.39 (tq, $J=7.4,1.2$ $\mathrm{Hz}, 2 \mathrm{H}), 0.94(\mathrm{t}, J=6.5 \mathrm{~Hz}, 18 \mathrm{H}), 0.82-0.66(2 \times \mathrm{q}, J=6.5 \mathrm{~Hz}, 2$ x 6H) $;{ }^{13} \mathrm{ppm} \mathrm{C} \mathrm{NMR}\left(126 \mathrm{MHz}, \mathrm{CDCl}_{3}\right) \delta=246.4,237.5,154.4$, 128.6, 48.8, 22.1, 7.4, 7.3, 3.9, 2.2 ppm; HRMS (ESI +ve) Exact mass calculated for $\mathrm{C}_{18} \mathrm{H}_{36} \mathrm{O}_{2} \mathrm{NaSi}_{2}[\mathrm{M}+\mathrm{Na}]^{+}: 363.2146$, found: 363.2150 .

\section{2-(Hydroxy(4-nitrophenyl)methyl)-1-(triethylsilyl)prop-2-en-}

1-one (6a): yield $88 \%$ as a yellow oil. FT-IR (film) 3464, 2956, 2876, 1594, 1519, 1344, 1014, 851, $737 \mathrm{~cm}^{-1} ;{ }^{1} \mathrm{H} \mathrm{NMR}(300 \mathrm{MHz}$, $\left.\mathrm{CDCl}_{3}\right) \delta=8.19(\mathrm{~d}, J=8.7 \mathrm{~Hz}, 2 \mathrm{H}), 7.53(\mathrm{~d}, J=8.7 \mathrm{~Hz}, 2 \mathrm{H}), 6.21$ $(\mathrm{s}, 1 \mathrm{H}), 6.17(\mathrm{~d}, J=1.1 \mathrm{~Hz}, 1 \mathrm{H}), 5.63(\mathrm{~d}, J=5.5 \mathrm{~Hz}, 1 \mathrm{H}), 3.37$ (dt, $J=5.2,2.7 \mathrm{~Hz}, 1 \mathrm{H}), 0.93(\mathrm{t}, J=7.5 \mathrm{~Hz}, 9 \mathrm{H}), 0.83-0.75(3 \times \mathrm{q}, J$ $=7.8 \mathrm{~Hz}, 6 \mathrm{H}) \mathrm{ppm} ;{ }^{13} \mathrm{C}$ NMR $\left(126 \mathrm{MHz}, \mathrm{CDCl}_{3}\right) \delta=239.6,154.3$, 149.1, 147.2, 129.6, 127.2, 123.5, 72.2, 7.2, 3.5 ppm; HRMS (ESI + ve) Exact mass calculated for $\mathrm{C}_{16} \mathrm{H}_{23} \mathrm{NO}_{4} \mathrm{NaSi}[\mathrm{M}+\mathrm{Na}]^{+}$: 344.1288, found: 344.1289 .

2-((4-Bromophenyl)(hydroxy)methyl)-1-(triethylsilyl)prop-2en-1-one (7a): yield $53 \%$ as a yellow oil. FT-IR (film) 3435, 2954, $2875,1590,1486,1010,731 \mathrm{~cm}^{-1} ;{ }^{1} \mathrm{H}$ NMR $\left(500 \mathrm{MHz}, \mathrm{CDCl}_{3}\right) \delta$ $=7.45(\mathrm{~d}, J=7.6 \mathrm{~Hz}, 2 \mathrm{H}), 7.22(\mathrm{~d}, J=7.7 \mathrm{~Hz}, 2 \mathrm{H}), 6.15(\mathrm{~s}, 1 \mathrm{H})$, $6.13(\mathrm{~d}, J=1.2 \mathrm{~Hz}, 1 \mathrm{H}), 5.51(\mathrm{~s}, 1 \mathrm{H}), 0.93(\mathrm{t}, J=7.5 \mathrm{~Hz}, 9 \mathrm{H}), 0.79$ (q $, J=7.5 \mathrm{~Hz}, 6 \mathrm{H}) \mathrm{ppm} ;{ }^{13} \mathrm{C} \mathrm{NMR}\left(126 \mathrm{MHz}, \mathrm{CDCl}_{3}\right) \delta=239.7$, $154.9,140.7,131.4,128.8,128.2,121.3,72.1,7.2$, 3.5 ppm; HRMS (ESI +ve) Exact mass calculated for $\mathrm{C}_{16} \mathrm{H}_{23} \mathrm{O}_{2} \mathrm{BrNaSi}$ $[\mathrm{M}+\mathrm{Na}]^{+}:$377.0542, found: 377.0543 .

2-((2-Bromophenyl)(hydroxy)methyl)-1-(triethylsilyl)prop-2en-1-one (8a): yield $63 \%$ as a yellow oil. FT-IR (film) 3065, 2960, 2912, 2876, 1596, 1466, 1020, $741 \mathrm{~cm}^{-1} ;{ }^{1} \mathrm{H}$ NMR $(500 \mathrm{MHz}$, $\left.\mathrm{CDCl}_{3}\right) \delta=7.59(\mathrm{dd}, J=8.0,1.7 \mathrm{~Hz}, 1 \mathrm{H}), 7.54(\mathrm{dd}, J=8.0,1.2$ $\mathrm{Hz}, 1 \mathrm{H}), 7.37$ (td, $J=7.6,1.2 \mathrm{~Hz}, 1 \mathrm{H}), 7.17(\mathrm{td}, J=7.6,1.7 \mathrm{~Hz}$, $1 \mathrm{H}), 6.12(\mathrm{~s}, 1 \mathrm{H}), 5.87(\mathrm{~d}, J=3.3 \mathrm{~Hz}, 1 \mathrm{H}), 5.80(\mathrm{~d}, J=1.0 \mathrm{~Hz}, 1 \mathrm{H})$, $3.52(\mathrm{~d}, J=3.7 \mathrm{~Hz}, 1 \mathrm{H}), 0.98(\mathrm{t}, J=7.2 \mathrm{~Hz}, 9 \mathrm{H}), 0.83-0.75(2 \mathrm{x}$ 
$q, J=7.8 \mathrm{~Hz}, 6 \mathrm{H}) \mathrm{ppm} ;{ }^{13} \mathrm{C} \mathrm{NMR}\left(126 \mathrm{MHz}, \mathrm{CDCl}_{3}\right) \delta=239.8$, 153.8, 140.0, 132.6, 129.5, 129.0, 128.5, 127.5, 122.6, 70.8, 7.3, $3.6 \mathrm{ppm}$; HRMS (ESI +ve) Exact mass calculated for $\mathrm{C}_{16} \mathrm{H}_{23} \mathrm{O}_{2} \mathrm{BrNaSi}[\mathrm{M}+\mathrm{Na}]^{+}: 377.0542$, found: 377.0542 .

2-((3-Bromophenyl)(hydroxy)methyl)-1-(triethylsilyl)prop-2en-1-one (9a): yield $75 \%$ as a yellow oil. FT-IR (film) 3435, 2954, $1875,1590,1486,1403,1234,1070,815,731 \mathrm{~cm}^{-1} ;{ }^{1} \mathrm{H}$ NMR $(400$ $\left.\mathrm{MHz}, \mathrm{CDCl}_{3}\right) \delta=7.47(\mathrm{~s}, 1 \mathrm{H}), 7.40-7.33(\mathrm{dd}, J=7.8,1.5 \mathrm{~Hz}, 1 \mathrm{H}$, $1 \mathrm{H}), 7.24$ (dd, $J=7.8,1.5 \mathrm{~Hz}, 1 \mathrm{H}), 7.17$ (td, $J=7.8,1.0 \mathrm{~Hz}, 1 \mathrm{H})$, 6.15-6.12 (m, 2H), $5.49(\mathrm{~d}, J=4.2 \mathrm{~Hz}, 1 \mathrm{H}), 3.49-3.42(\mathrm{~m}, 1 \mathrm{H})$, $0.91(\mathrm{t}, J=7.8 \mathrm{~Hz}, 9 \mathrm{H}), 0.77(\mathrm{q}, J=7.8 \mathrm{~Hz}, 6 \mathrm{H}) \mathrm{ppm} ;{ }^{13} \mathrm{C} \mathrm{NMR}$ $\left(101 \mathrm{MHz}, \mathrm{CDCl}_{3}\right) \delta=239.7,154.8,144.1,130.5,129.8,129.5$, 128.8, 125.2, 122.4, 71.9, 7.2, 3.5 ppm; HRMS (ESI +ve) Exact mass calculated for $\mathrm{C}_{16} \mathrm{H}_{23} \mathrm{O}_{2} \mathrm{BrNaSi}[\mathrm{M}+\mathrm{Na}]^{+}: 377.0542$, found: 377.0542 .

2-(Hydroxy(pyridin-3-yl)methyl)-1-(triethylsilyl)prop-2-en-1one (10a): yield $68 \%$ as a yellow oil. FT-IR (film) 3170, 2954, $2875,1593,1424,948,711 \mathrm{~cm}^{-1} ;{ }^{1} \mathrm{H}$ NMR $\left(300 \mathrm{MHz}, \mathrm{CDCl}_{3}\right) \delta=$ $8.46(\mathrm{~d}, 0.9 \mathrm{~Hz}, 1 \mathrm{H}), 8.38$ (dd, $J=4.8,1.9 \mathrm{~Hz}, 1 \mathrm{H}), 7.68$ (ddt, $J=$ 7.8, 1.9, $0.9 \mathrm{~Hz}, 1 \mathrm{H}), 7.21$ (dd, $J=7.8,4.8, \mathrm{~Hz}, 1 \mathrm{H}), 6.27(\mathrm{~s}, 1 \mathrm{H})$, $6.18(\mathrm{~s}, 1 \mathrm{H}), 5.58(\mathrm{~s}, 1 \mathrm{H}), 0.88(\mathrm{t}, J=6.9,9 \mathrm{H}), 0.77(\mathrm{~m}, 6 \mathrm{H}) \mathrm{ppm}$; ${ }^{13} \mathrm{C} \mathrm{NMR}\left(126 \mathrm{MHz}, \mathrm{CDCl}_{3}\right) \delta=238.2,155.5,148.1,147.9,138.4$, 134.7, 128.1, 123.2, 68.9, 7.1, 3.5 ppm; HRMS (ESI +ve) Exact mass calculated for $\mathrm{C}_{15} \mathrm{H}_{23} \mathrm{NO}_{2} \mathrm{NaSi}[\mathrm{M}+\mathrm{Na}]+: 300.1390$, found: 300.1390 .

Ethyl 2-hydroxy-3-((triethylsilyl)carbonyl)but-3-enoate (11a): yield $93 \%$ as a yellow oil. FT-IR (film) 3492, 2956, 2877, 1736, 1598, 1234, 1086, 1019, $735 \mathrm{~cm}^{-1} ;{ }^{1} \mathrm{H}$ NMR $\left(400 \mathrm{MHz}, \mathrm{CDCl}_{3}\right) \delta$ $=6.31(\mathrm{~s}, 1 \mathrm{H}), 6.19(\mathrm{~s}, 1 \mathrm{H}), 4.79(\mathrm{~d}, J=6.3 \mathrm{~Hz}, 1 \mathrm{H}), 4.19(\mathrm{q}, J=$ $7.0 \mathrm{~Hz}, 2 \mathrm{H}), 3.38(\mathrm{~d}, J=6.3 \mathrm{~Hz}, 1 \mathrm{H}), 1.21(\mathrm{t}, J=7.0 \mathrm{~Hz}, 3 \mathrm{H}), 0.97$ (t, $J=8.2,9 \mathrm{H}), 0.81$ (q, $J=8.2 \mathrm{~Hz}, 6 \mathrm{H}) \mathrm{ppm} ;{ }^{13} \mathrm{C} \mathrm{NMR}(101 \mathrm{MHz}$, $\left.\mathrm{CDCl}_{3}\right) \delta=236.2,172.6,151.3,129.9,69.9,61.7,13.9,7.1,3.4$ ppm; HRMS (ESI +ve) Exact mass calculated for $\mathrm{C}_{13} \mathrm{H}_{24} \mathrm{O}_{4} \mathrm{NaSi}$ $[\mathrm{M}+\mathrm{Na}]^{+}:$295.1336, found: 295.1335 .

3-Hydroxy-2-methylene-1-(triethylsilyl)heptan-1-one (12a): yield $37 \%$ as a yellow oil. FT-IR (film) $3435,2955,2875,1592$, $1414,1017,731 \mathrm{~cm}^{-1} ;{ }^{1} \mathrm{H}$ NMR $\left(300 \mathrm{MHz}, \mathrm{CDCl}_{3}\right) \delta=6.20(\mathrm{~s}, 1 \mathrm{H})$, $6.05(\mathrm{~s}, 1 \mathrm{H}), 4.39(\mathrm{q}, J=6.4 \mathrm{~Hz}, 1 \mathrm{H}), 1.60-1.53(\mathrm{~m}, 2 \mathrm{H}), 1.42-1.23$ (m, 4H), 0.98 (t, $J=8.2 \mathrm{~Hz}, 9 \mathrm{H}$ ), 0.90 (t, $J=7.0 \mathrm{~Hz}, 3 \mathrm{H}$ ), 0.81 (q, $J=8.2 \mathrm{~Hz}, 6 \mathrm{H}) \mathrm{ppm} ;{ }^{13} \mathrm{C} \mathrm{NMR}\left(126 \mathrm{MHz}, \mathrm{CDCl}_{3}\right) \delta=240.1,155.9$, 127.7, 71.2, 35.9, 28.0, 22.5, 14.0, 7.3, 3.7 ppm; HRMS (ESI +ve) Exact mass calculated for $\mathrm{C}_{14} \mathrm{H}_{28} \mathrm{O}_{2} \mathrm{NaSi}[\mathrm{M}+\mathrm{Na}]^{+}:$279.1750, found: 279.1753 .

1-(Dimethyl(phenyl)silyl)-2-(hydroxy(phenyl)methyl)prop-2en-1-one (4b): yield $40 \%$ as a yellow oil. FT-IR (film) 3493, 2959, $1597,1427,1251,1112,1058,814,781,695,650 \mathrm{~cm}^{-1} ;{ }^{1} \mathrm{H}$ NMR $\left(300 \mathrm{MHz}, \mathrm{CDCl}_{3}\right) \delta=7.51-7.46(\mathrm{~m}, 2 \mathrm{H}), 7.44-7.37(\mathrm{~m}, 3 \mathrm{H})$, 7.34$7.26(\mathrm{~m}, 5 \mathrm{H}), 6.05(\mathrm{~s}, 1 \mathrm{H}), 6.02(\mathrm{~d}, J=1.2 \mathrm{~Hz}, 1 \mathrm{H}), 5.55(\mathrm{~d}, J=$ $4.7 \mathrm{~Hz}, 1 \mathrm{H}), 3.08(\mathrm{~d}, J=5.0 \mathrm{~Hz}, 1 \mathrm{H}), 0.53$ and 0.51 (s, 3H) ppm; ${ }^{13} \mathrm{C}$ NMR $\left(75 \mathrm{MHz}, \mathrm{CDCl}_{3}\right) \delta 237.4,153.9,141.4,135.3,133.8$, 130.2, 129.9, 128.3, 128.2, 127.5, 126.5, 72.5, -3.1, -3.2 ppm; HRMS (ESI +ve) Exact mass calculated for $\mathrm{C}_{18} \mathrm{H}_{20} \mathrm{O}_{2} \mathrm{NaSi}$ $[\mathrm{M}+\mathrm{Na}]^{+}:$319.1130, found: 319.1134 .
1,5-Bis(dimethyl(phenyl)silyl)-2-methylenepentane-1,5-dione (5b): yield $11 \%$ as a yellow oil. FT-IR (film) 2960, 1642, 1596, 1427, 1251, 1109, 812, 781, 730, 697, $649 \mathrm{~cm}^{-1} ;{ }^{1} \mathrm{H}$ NMR $(300$ $\left.\mathrm{MHz}, \mathrm{CDCl}_{3}\right) \delta=7.50(\mathrm{~m}, 4 \mathrm{H}), 7.36(\mathrm{~m}, 6 \mathrm{H}), 5.81(\mathrm{~d}, J=2.3 \mathrm{~Hz}$, $2 \mathrm{H}), 2.63(\mathrm{t}, J=7.2 \mathrm{~Hz}, 2 \mathrm{H}), 2.34(\mathrm{t}, J=7.2 \mathrm{~Hz}, 2 \mathrm{H}), 0.49$ and 0.45 (s, 6H) ppm; ${ }^{13} \mathrm{C} \mathrm{NMR}\left(75 \mathrm{MHz}, \mathrm{CDCl}_{3}\right) \delta=244.5,235.2$, 152.5, 136.0, 134.3, 133.9, 133.8, 130.0, 129.9, 129.7, 128.2, 47.2, 22.2, -2.7, -4.7 ppm; HRMS (ESI +ve) Exact mass calculated for $\mathrm{C}_{22} \mathrm{H}_{28} \mathrm{O}_{2} \mathrm{NaSi}_{2} \quad[\mathrm{M}+\mathrm{Na}]^{+}: 403.1526$, found: 403.1522 .

1-(Dimethyl(phenyl)silyl)-2-(hydroxy(4-nitrophenyl)methyl) prop-2-en-1-one (6b): yield $77 \%$ as a yellow oil. FT-IR (film) 3486, 2959, 1688, 1596, 1518, 1344, 1252, 1109, 1066, 818, 786, 735, $652 \mathrm{~cm}^{-1} ;{ }^{1} \mathrm{H}$ NMR $\left(300 \mathrm{MHz}, \mathrm{CDCl}_{3}\right) \delta=7.95(\mathrm{~d}, J=8.7 \mathrm{~Hz}, 2 \mathrm{H})$, 7.29 - $7.24(\mathrm{~m}, 4 \mathrm{H}), 7.23$ - $7.13(\mathrm{~m}, 3 \mathrm{H}), 5.92(\mathrm{~s}, 1 \mathrm{H}), 5.89(\mathrm{~s}, 1 \mathrm{H})$, $5.39(\mathrm{~d}, J=5.4 \mathrm{~Hz}, 1 \mathrm{H}), 3.15(\mathrm{~d}, J=5.4,1 \mathrm{H}), 0.33(\mathrm{~s}, 6 \mathrm{H}) \mathrm{ppm}$; ${ }^{13} \mathrm{C}$ NMR $\left(75 \mathrm{MHz}, \mathrm{CDCl}_{3}\right) \delta=237.3,152.9,148.9,147.2,134.7$, 133.7, 130.9, 130.1, 128.3, 127.2, 123.5, 72.0 -3.2 ppm; HRMS $(\mathrm{ESI}+\mathrm{ve})$ Exact mass calculated for $\mathrm{C}_{18} \mathrm{H}_{19} \mathrm{NO}_{4} \mathrm{NaSi}[\mathrm{M}+\mathrm{Na}]^{+}$: 364.0981, found: 364.0981 .

\section{2-((4-Bromophenyl)(hydroxy)methyl)-1-(dimethyl(phenyl)}

silyl)prop-2-en-1-one (7b): yield $34 \%$ as a yellow oil. FT-IR (film) 3449, 2959, 1690, 1593, 1485, 1252, 1112, 1069, 1009, 818, 734, $652 \mathrm{~cm}^{-1} ;{ }^{1} \mathrm{H}$ NMR $\left(300 \mathrm{MHz}, \mathrm{CDCl}_{3}\right) \delta=7.49-7.30(\mathrm{~m}, 7 \mathrm{H}), 7.17-$ $7.10(\mathrm{~d}, J=8.7 \mathrm{~Hz}, 2 \mathrm{H}), 6.03(\mathrm{~s}, 1 \mathrm{H}), 6.00(\mathrm{~d}, J=1.2 \mathrm{~Hz}, 1 \mathrm{H})$, $5.45(\mathrm{~s}, 1 \mathrm{H}), 3.18(\mathrm{~s}, 1 \mathrm{H}), 0.50(\mathrm{~s}, 3 \mathrm{H}), 0.49(\mathrm{~s}, 3 \mathrm{H}) \mathrm{ppm} ;{ }^{13} \mathrm{C}$ NMR $\left(75 \mathrm{MHz}, \mathrm{CDCl}_{3}\right) \delta=234.8,150.9,137.9,132.5,131.2,128.8$, 127.7, 127.4, 125.7, 125.7, 118.9, 69.4, -5.6, -5.7 ppm; HRMS $(\mathrm{ESI}+\mathrm{ve})$ Exact mass calculated for $\mathrm{C}_{18} \mathrm{H}_{19} \mathrm{O}_{2} \mathrm{NaSiBr}[\mathrm{M}+\mathrm{Na}]^{+}$: 397.0235, found: 397.0241 .

Ethyl-3-((dimethyl(phenyl)silyl)carbonyl)-2-hydroxybut-3enoate (11b): yield $88 \%$ as a yellow oil. FT-IR (film) 3491,2853 , $1608,1428,1249,1029,827,733,647 \mathrm{~cm}^{-1} ;{ }^{1} \mathrm{H}$ NMR $(300 \mathrm{MHz}$, $\left.\mathrm{CDCl}_{3}\right) \delta=7.59-7.49(\mathrm{~m}, 2 \mathrm{H}), 7.45-7.33(\mathrm{~m}, 3 \mathrm{H}), 6.20(\mathrm{~s}, 1 \mathrm{H})$, 6.06, (s, 1H), $4.79(\mathrm{~d}, J=4.2 \mathrm{~Hz}, 1 \mathrm{H}), 4.29-4.05(\mathrm{~m}, 2 \mathrm{H}), 3.43(\mathrm{~d}$, $J=5.8 \mathrm{~Hz}, 1 \mathrm{H}), 1.20(\mathrm{t}, J=7.1 \mathrm{~Hz}, 3 \mathrm{H}), 0.56(\mathrm{~s}, 3 \mathrm{H}), 0.54(\mathrm{~s}, 3 \mathrm{H})$ ppm; ${ }^{13} \mathrm{C}$ NMR $\left(75 \mathrm{MHz}, \mathrm{CDCl}_{3}\right) \delta=234.4,172.6,149.9,135.2$, $133.8,131.5,129.9,128.3,70.1,61.9,14.0,-3.1,-3.2$ ppm; HRMS (ESI +ve) Exact mass calculated for $\mathrm{C}_{15} \mathrm{H}_{20} \mathrm{O}_{4} \mathrm{NaSi}$ $[\mathrm{M}+\mathrm{Na}]^{+}:$315.1029, found: 315.1032 .

\section{2-(Hydroxy(4-nitrophenyl)methyl)-1-(trimethylsilyl)prop-2-}

en-1-one (6c): yield 92\% as a yellow oil. FT-IR (film) 2960, 1596, $1518,1410,1343,1249,1014,986,839,755,700,624 \mathrm{~cm}^{-1} ;{ }^{1} \mathrm{H}$ $\operatorname{NMR}\left(300 \mathrm{MHz}, \mathrm{CDCl}_{3}\right) \delta=8.17(\mathrm{~d}, J=8.8 \mathrm{~Hz}, 2 \mathrm{H}), 7.48(\mathrm{~d}, J=$ $8.8 \mathrm{~Hz}, 2 \mathrm{H}), 6.24(\mathrm{~s}, 1 \mathrm{H}), 6.18(\mathrm{~d}, J=1.1 \mathrm{~Hz}, 1 \mathrm{H}), 5.59(\mathrm{~d}, J=$ $4.50 \mathrm{~Hz}, 1 \mathrm{H}), 0.26(\mathrm{~s}, 9 \mathrm{H}) \mathrm{ppm} ;{ }^{13} \mathrm{C}$ NMR $\left(75 \mathrm{MHz}, \mathrm{CDCl}_{3}\right) \delta=$ 239.2, 153.0, 149.0, 147.2, 130.2, 127.2, 123.5, 71.9, -1.5 ppm; HRMS (ESI +ve) Exact mass calculated for $\mathrm{C}_{13} \mathrm{H}_{17} \mathrm{NO}_{4} \mathrm{NaSi}$ $[\mathrm{M}+\mathrm{H}]+:$ 302.0819, found: 302.0818 .

\section{Ethyl-2-hydroxy-3-((trimethylsilyl)carbonyl)but-3-enoate}

(11c): yield $22 \%$ as a yellow oil. FT-IR (film) 3451, 2982, 2930, 1742, 1701, 1451, 1372, 1207, 1020, 759, $691 \mathrm{~cm}^{-1} ;{ }^{1} \mathrm{H}$ NMR $(300$ $\left.\mathrm{MHz}, \mathrm{CDCl}_{3}\right) \delta=6.27(\mathrm{~d}, J=0.9 \mathrm{~Hz}, 1 \mathrm{H}), 6.16(\mathrm{~s}, 1 \mathrm{H}), 4.78(\mathrm{~d}, J$ $=5.2 \mathrm{~Hz}, 1 \mathrm{H}), 4.29-3.99(\mathrm{~m}, 2 \mathrm{H}), 3.44(\mathrm{~d}, J=6.2 \mathrm{~Hz}, 1 \mathrm{H}), 1.18$ 
(t, $J=7.1 \mathrm{~Hz}, 3 \mathrm{H}), 0.25$ (s, 9H) ppm; ${ }^{13} \mathrm{C} \mathrm{NMR}\left(75 \mathrm{MHz}, \mathrm{CDCl}_{3}\right) \delta$ = 236.3, 172.6, 150.1, 129.9, 70.1, 61.7, 14.0, -1.7 ppm; HRMS $(\mathrm{ESI}+\mathrm{ve})$ Exact mass calculated for $\mathrm{C}_{10} \mathrm{H}_{18} \mathrm{O}_{4} \mathrm{Na} \mathrm{Si}[\mathrm{M}+\mathrm{H}]+$ : 253.0866, found: 253.0866 .

2-(Hydroxy(phenyl)methyl)-1-(triisopropylsilyl)prop-2-en-1one (4d): yield 10\% as a yellow oil. FT-IR (film) 3493, 2944, 2894, $1659,1465,1113,992,882,751 \mathrm{~cm}^{-1} ;{ }^{1} \mathrm{H}$ NMR $\left(500 \mathrm{MHz}, \mathrm{CDCl}_{3}\right)$ $\delta=7.35-7.30(\mathrm{~m}, 3 \mathrm{H}), 7.27-7.23(\mathrm{~m}, 2 \mathrm{H}), 6.10(\mathrm{~d}, J=1.0 \mathrm{~Hz}, 1 \mathrm{H})$, $6.05(\mathrm{~s}, 1 \mathrm{H}), 5.58(\mathrm{~s}, 1 \mathrm{H}), 3.34-3.01(\mathrm{~m}, 1 \mathrm{H}), 1.30-1.20(\mathrm{~m}, 3 \mathrm{H})$, $095(\mathrm{~s}, J=6.2 \mathrm{~Hz}, 9 \mathrm{H}), 0.98(\mathrm{~s}, J=6.2 \mathrm{~Hz}, 9 \mathrm{H}) \mathrm{ppm} ;{ }^{13} \mathrm{C} \mathrm{NMR}$ $\left(75 \mathrm{MHz}, \mathrm{CDCl}_{3}\right) \delta=239.6,156.3,141.5,128.2,127.5,126.6$, 73.0, 29.7, 18.5, $12.2 \mathrm{ppm}$; HRMS (ESI +ve) Exact mass calculated for $\mathrm{C}_{19} \mathrm{H}_{31} \mathrm{O}_{2} \mathrm{Si}[\mathrm{M}+\mathrm{H}]^{+}:$319.2093, found: 319.2086 .

2-methylene-1,5-bis(triisopropylsilyl)pentane-1,5-dione (5d): yield $20 \%$ as a yellow oil. FT-IR (film) 2944, 2867, 1639, 1594, 1464, 1256, 1168, 922, 882, $675 \mathrm{~cm}^{-1} ;{ }^{1} \mathrm{H}$ NMR $\left(300 \mathrm{MHz}, \mathrm{CDCl}_{3}\right)$ $\delta=6.02(\mathrm{~s}, 1 \mathrm{H}), 5.82(\mathrm{~s}, 1 \mathrm{H}), 2.60(\mathrm{t}, J=7.1 \mathrm{~Hz}, 2 \mathrm{H}), 2.36(\mathrm{t}, J=$ $7.1 \mathrm{~Hz}, 2 \mathrm{H}), 1.41-1.18(\mathrm{~m}, 6 \mathrm{H}), 1.07(\mathrm{~d}, J=2.6 \mathrm{~Hz}, 18 \mathrm{H}), 1.04(\mathrm{~d}$, $J=2.6 \mathrm{~Hz}, 18 \mathrm{H}) \mathrm{ppm} ;{ }^{13} \mathrm{C}$ NMR $\left(75 \mathrm{MHz}, \mathrm{CDCl}_{3}\right) \delta=245.5,236.8$, 155.2, 128.1, 50.1, 22.1, 18.7, 18.5, 12.3, 10.7 ppm; HRMS: (ESI +ve) Exact mass calculated for $\mathrm{C}_{24} \mathrm{H}_{48} \mathrm{O}_{2} \mathrm{NaSi}_{2}[\mathrm{M}+\mathrm{Na}]^{+}: 447.3091$ found: 447.3084 .

2-(Hydroxy(4-nitrophenyl)methyl)-1-(triisopropylsilyl)prop-2en-1-one (6d): yield $30 \%$ as a yellow oil. FT-IR (film) 3486, 2944, 2892, 1600, 1465, 1250, 1177, 1015, 920, 882, 677, $642 \mathrm{~cm}^{-1} ;{ }^{1} \mathrm{H}$ $\operatorname{NMR}\left(300 \mathrm{MHz}, \mathrm{CDCl}_{3}\right) \delta=8.02(\mathrm{~d}, J=8.8 \mathrm{~Hz}, 2 \mathrm{H}), 7.37(\mathrm{~d}, J=$ $8.8 \mathrm{~Hz}, 2 \mathrm{H}), 6.0(\mathrm{~s}, 1 \mathrm{H}), 5.97(\mathrm{~s}, 1 \mathrm{H}), 5.47(\mathrm{~d}, J=5.5 \mathrm{~Hz}, 1 \mathrm{H})$, $3.21(\mathrm{~d}, J=5.6 \mathrm{~Hz}, 1 \mathrm{H}), 1.22-1.11(\mathrm{~m}, 3 \mathrm{H}), 0.88(\mathrm{~d}, J=7.5 \mathrm{~Hz}$, $9 \mathrm{H}), 0.86(\mathrm{~d}, J=7.5 \mathrm{~Hz}, 9 \mathrm{H}) \mathrm{ppm} ;{ }^{13} \mathrm{C}$ NMR $\left(75 \mathrm{MHz}, \mathrm{CDCl}_{3}\right) \delta=$ 236.8, 152.5, 146.6, 144.6, 126.8, 124.7, 120.9, 69.8, 16.0, 9.6 ppm; HRMS (ESI -ve) Exact mass calculated for $\mathrm{C}_{19} \mathrm{H}_{30} \mathrm{NO}_{4} \mathrm{Si}$ $[\mathrm{M}+\mathrm{H}]^{+}:$364.1944, found: 364.1947 .

Ethyl -2-hydroxy-3-((triisopropylsilyl)carbonyl)but-3-enoate (11d): yield $70 \%$ as a yellow oil. FT-IR (film) 3491, 2944, 1602, $1371,1190,1026,857,741,635 \mathrm{~cm}^{-1} ;{ }^{1} \mathrm{H}$ NMR $\left(300 \mathrm{MHz}, \mathrm{CDCl}_{3}\right)$ $\delta=6.33(\mathrm{~s}, 1 \mathrm{H}), 6.16(\mathrm{~s}, 1 \mathrm{H}), 4.77(\mathrm{~d}, J=6.4 \mathrm{~Hz}, 1 \mathrm{H}), 4.18(\mathrm{q}, J$ $=7.2 \mathrm{~Hz}, 2 \mathrm{H}), 3.41(\mathrm{~d}, J=6.5 \mathrm{~Hz}, 1 \mathrm{H}), 1.44-1.27(\mathrm{~m}, 3 \mathrm{H}), 1.20(\mathrm{t}$, $J=7.2 \mathrm{~Hz}, 3 \mathrm{H}), 1.10(\mathrm{dd}, J=7.5,1.2 \mathrm{~Hz}, 18 \mathrm{H}) \mathrm{ppm} ;{ }^{13} \mathrm{C} \mathrm{NMR}$ $\left(75 \mathrm{MHz}, \mathrm{CDCl}_{3}\right) \delta=235.8,172.8,151.9,130.3,70.5,61.8,18.6$, 13.9, $12.2 \mathrm{ppm}$; HRMS (ESI +ve) Exact mass calculated for $\mathrm{C}_{16} \mathrm{H}_{30} \mathrm{O}_{4} \mathrm{NaSi}[\mathrm{M}+\mathrm{Na}]^{+}:$337.1811, found: 337.1811.

\section{1-(Tert-butyldimethylsilyl)-2-(hydroxy(4-nitrophenyl)methyl)} prop-2-en-1-one (6e): yield $34 \%$ as a yellow oil. FT-IR (film) 3480 , 2930, 28602, 1590, 1520,1345, 1250, 821, $778 \mathrm{~cm}^{-1}$; ${ }^{1} \mathrm{H}$ NMR $\left(300 \mathrm{MHz}, \mathrm{CDCl}_{3}\right) \delta=8.18(\mathrm{~d}, J=8.8 \mathrm{~Hz}, 2 \mathrm{H}), 7.53(\mathrm{~d}, J=8.8 \mathrm{~Hz}$, 2H), $6.22(\mathrm{~d}, J=0.5 \mathrm{~Hz}, 1 \mathrm{H}), 6.18(\mathrm{~d}, J=1.1 \mathrm{~Hz}, 1 \mathrm{H}), 5.63(\mathrm{~d}, J$ $=4.1 \mathrm{~Hz}, 1 \mathrm{H}), 3.42(\mathrm{~d}, J=5.2 \mathrm{~Hz}, 1 \mathrm{H}), 0.86(\mathrm{~s}, 9 \mathrm{H}), 0.26(\mathrm{~s}, 3 \mathrm{H})$, $0.25(\mathrm{~s}, 3 \mathrm{H}) \mathrm{ppm} ;{ }^{13} \mathrm{C} \mathrm{NMR}\left(75 \mathrm{MHz}, \mathrm{CDCl}_{3}\right) \delta=247.5,238.9$, 154.6, 149.1, 147.2, 130.3, 127.2, 123.49, 72.1, 16.7, -4.8 ppm; HRMS (ESI +ve) Exact mass calculated for $\mathrm{C}_{16} \mathrm{H}_{23} \mathrm{NO}_{4} \mathrm{NaSi}$ $[\mathrm{M}+\mathrm{Na}]^{+}:$344.1288, found: 344.1289 .

Ethyl-3-((tert-butyldimethylsilyl)carbonyl)-2-hydroxybut-3enoate (11e): yield 54\% as a yellow oil. FT-IR (film) 3445, 2929,
$2857,1743,1253,1089,1040,840,779 \mathrm{~cm}^{-1} ;{ }^{1} \mathrm{H}$ NMR $(300 \mathrm{MHz}$, $\left.\mathrm{CDCl}_{3}\right) \delta=6.33(\mathrm{~s}, 1 \mathrm{H}), 6.22(\mathrm{~s}, 1 \mathrm{H}), 4.76(\mathrm{~d}, J=6.1 \mathrm{~Hz}, 1 \mathrm{H})$, 4.21 (q, $J=7.1 \mathrm{~Hz}, 2 \mathrm{H}$ ), 3.37 (d, $J=6.0 \mathrm{~Hz}, 1 \mathrm{H}), 1.24$ (t, $J=7.1$ $\mathrm{Hz}, 3 \mathrm{H}), 0.94$ (s, 9H), 0.30 (s, 3H), 0.29 (s, 3H) ppm; ${ }^{13} \mathrm{C}$ NMR $(75$ $\left.\mathrm{MHz}, \mathrm{CDCl}_{3}\right) \delta=235.9,172.8,151.4,131.1,70.6,61.9,26.5,16.8$, 14.0, -4.9 ppm; HRMS (ESI +ve) Exact mass calculated for $\mathrm{C}_{13} \mathrm{H}_{24} \mathrm{O}_{4} \mathrm{NaSi}[\mathrm{M}+\mathrm{Na}]^{+}:$295.1341, found: 295.1339.

2-(Hydroxy(phenyl)methyl)-1-(methyldiphenylsilyl)prop-2en-1-one (6f): yield $72 \%$ as a yellow oil. FT-IR (film) 3070, 1688, 1596, 1518, 1428, 1344, 1109, 792, 728, $696 \mathrm{~cm}^{-1} ;{ }^{1} \mathrm{H}$ NMR $(300$ $\left.\mathrm{MHz}, \mathrm{CDCl}_{3}\right) \delta=8.13(\mathrm{~d}, J=8.7 \mathrm{~Hz}, 2 \mathrm{H}), 7.55-7.31(\mathrm{~m}, 12 \mathrm{H})$, $6.15(\mathrm{~s}, 1 \mathrm{H}), 6.11(\mathrm{~s}, 1 \mathrm{H}), 5.67(\mathrm{~s}, 1 \mathrm{H}), 3.60(\mathrm{~s}, 1 \mathrm{H}), 0.80(\mathrm{~s}, 3 \mathrm{H})$ ppm; ${ }^{13} \mathrm{C} \mathrm{NMR}\left(75 \mathrm{MHz}, \mathrm{CDCl}_{3}\right) \delta=235.3,153.7,149.2,147.2$, 135.0, 134.9, 134.0, 133.0, 132.9, 131.7, 130.3, 128.4, 128.3, 127.3, 123.5, 71.7, -3.6 ppm; HRMS (ESI +ve) Exact mass calculated for $\mathrm{C}_{23} \mathrm{H}_{21} \mathrm{NO}_{4} \mathrm{NaSi}[\mathrm{M}+\mathrm{Na}]^{+}:$426.1132, found: 426.1129 .

\section{Ethyl-2-hydroxy-3-((methyldiphenylsilyl)carbonyl)but-3-}

enoate (11f): yield $32 \%$ as a yellow oil. FT-IR (film) 3450,3070 , 2981, 1734, 1694, 1428, 1254, 1112, 1024, 790, 724, $697 \mathrm{~cm}^{-1}$; ${ }_{1}^{1} \mathrm{H} \mathrm{NMR}\left(300 \mathrm{MHz}, \mathrm{CDCl}_{3}\right) \delta=7.62-7.56(\mathrm{~m}, 4 \mathrm{H}), 7.48-7.32(\mathrm{~m}$, $6 \mathrm{H}), 6.24(\mathrm{~s}, 1 \mathrm{H}), 6.10(\mathrm{~s}, 1 \mathrm{H}), 4.86(\mathrm{~s}, 1 \mathrm{H}), 4.20(\mathrm{~m}, 2 \mathrm{H}), 3.55$ $(\mathrm{d}, J=5.6 \mathrm{~Hz}, 1 \mathrm{H}), 1.19(\mathrm{t}, J=7.1 \mathrm{~Hz}, 3 \mathrm{H}), 0.81(\mathrm{~s}, 3 \mathrm{H}) \mathrm{ppm} ;{ }^{13} \mathrm{C}$ NMR $\left(75 \mathrm{MHz}, \mathrm{CDCl}_{3}\right) \delta=232.7,172.6,150.5,135.0,133.3$, 133.2, 132.7, 130.2, 128.3, 70.2, 62.0, 14.0, -3.6 ppm; HRMS $(\mathrm{ESI}+\mathrm{ve})$ Exact mass calculated for $\mathrm{C}_{20} \mathrm{H}_{22} \mathrm{O}_{4} \mathrm{NaSi}[\mathrm{M}+\mathrm{Na}]^{+}$: 377.1179, found: 377.1177 .

\section{General experimental procedure for the protection of $\mathrm{MBH}$ adducts as acetates:}

Three methods have to been employed to protect the previous $\mathrm{MBH}$ products. After some time we found that the use of TEA was not necessary and induced the formation of transposed product. Finally $\mathrm{NaHCO}_{3}$ was found to be more efficient for sensitive substrates. We described the three methods which were used:

Method A: a round bottom flask was charged with $\beta$ hydroxyacryloylsilane (1 equiv), acetic anhydride ( 2 equiv), in dry DCM under nitrogen flush at $0{ }^{\circ} \mathrm{C}$. Then DMAP ( 0.1 equiv) and TEA ( 3 equiv) were added to the solution and stirring was continued at room temperature and monitored by TLC using 10\% EtOAc/pentane. After aqueous workup and concentration, the compound was purified by column chromatography.

Method B: a round bottom flask was charged with $\beta$ hydroxyacryloylsilane (1 equiv), acetic anhydride $(1,2-1,5$ equiv) in dry DCM under nitrogen flush at $0{ }^{\circ} \mathrm{C}$. Then DMAP ( 0.2 equiv) was added to the solution and stirring was continued at room temperature and monitored by TLC using $10 \%$ EtOAc/pentane. After aqueous workup and concentration, the compound was purified by column chromatography.

Method C: a round bottom flask was charged with $\beta$ hydroxyacryloylsilane ( 1 equiv), acetic anhydride (5 equiv) in dried DCM under nitrogen flush at $0^{\circ} \mathrm{C}$. Then, $\mathrm{NaHCO}_{3}$ (2.0 equiv) was added to the solution and stirring was continued at room temperature and the reaction monitored by TLC using $10 \%$ 
EtOAc/pentane. After aqueous workup and concentration, the compound was purified by column chromatography.

1-Phenyl-2-((triethylsilyl)carbonyl)allylacetate (13a): method A: yield $63 \%$ as a yellow oil. FT-IR (film) 2955, 2876, 1740, 1599, $1369,1223,1026,696 \mathrm{~cm}^{-1} ;{ }^{1} \mathrm{H}$ NMR $\left(500 \mathrm{MHz}, \mathrm{CDCl}_{3}\right) \delta=7.38-$ $7.24(\mathrm{~m}, 5 \mathrm{H}), 6.69(\mathrm{~s}, 1 \mathrm{H}), 6.23(\mathrm{~d}, J=1.5 \mathrm{~Hz}, 1 \mathrm{H}), 6.16(\mathrm{~s}, 1 \mathrm{H})$, 2.10 (s, 3H), 0.92 (t, $J=7.8 \mathrm{~Hz}, 9 \mathrm{H}), 0.78$ (q, $J=7.7 \mathrm{~Hz}, 6 \mathrm{H}) \mathrm{ppm}$; ${ }^{13} \mathrm{C}$ NMR $\left(126 \mathrm{MHz}, \mathrm{CDCl}_{3}\right) \delta=235.5,169.3,153.4,138.4,128.3$, 128.0, 127.5, 126.6, 72.1, 21.1, 7.2, 3.5 ppm; HRMS (ESI +ve) Exact mass calculated for $\mathrm{C}_{18} \mathrm{H}_{26} \mathrm{O}_{3} \mathrm{NaSi}[\mathrm{M}+\mathrm{Na}]^{+}: 341.1543$, found: 341.1545 .

1-(4-Nitrophenyl)-2-((triethylsilyl)carbonyl)allyl acetate (14a): method $A$ : yield $92 \%$ as a yellow thick oil. When time was prolonged from $6 \mathrm{~h}$ to $48 \mathrm{~h}$ isomerisation of $14 \mathrm{a}$ to 14 'a took place and this compound (14'a, $E$ isomer) was obtained in $18 \%$ yield together with compound (14a) in $81 \%$. Method C: yield: $82 \%$ as a yellow solid. FT-IR (film) 2596, 2876, 1745, 1598, 1521, 1345, 1220, 735, $698 \mathrm{~cm}^{-1} ;{ }^{1} \mathrm{H} \mathrm{NMR}\left(300 \mathrm{MHz}, \mathrm{CDCl}_{3}\right) \delta 8.18$ (d, $J=8.7$ $\mathrm{Hz}, 2 \mathrm{H}), 7.53(\mathrm{~d}, J=8.7 \mathrm{~Hz}, 2 \mathrm{H}), 6.69(\mathrm{~s}, 1 \mathrm{H}), 6.34(\mathrm{~d}, J=1.4 \mathrm{~Hz}$, $1 \mathrm{H}), 6.25(\mathrm{~s}, 1 \mathrm{H}), 2.12(\mathrm{~s}, 3 \mathrm{H}), 0.96-0.84(\mathrm{~m}, 9 \mathrm{H}), 0.81-0.71$ $(\mathrm{m}, 6 \mathrm{H}) \mathrm{ppm} ;{ }^{13} \mathrm{C}$ NMR $\left(75 \mathrm{MHz}, \mathrm{CDCl}_{3}\right) \delta 235.3,169.1,152.3$, 147.4, 145.8, 128.2, 127.9, 123.6, 71.1, 21.0, 7.2, 3.5 ppm; HRMS $(\mathrm{ESI}+\mathrm{ve})$ Exact mass calculated for $\mathrm{C}_{18} \mathrm{H}_{25} \mathrm{NO}_{5} \mathrm{NaSi}[\mathrm{M}+\mathrm{Na}]^{+}$: 386.1394, found: 386.1390 .

(E)-3-(4-Nitrophenyl)-2-((triethylsilyl)carbonyl)allyl acetate (14a'): ${ }^{1} \mathrm{H}$ NMR $\left(300 \mathrm{MHz}, \mathrm{CDCl}_{3}\right) \delta=8.30(\mathrm{~d}, J=8.7 \mathrm{~Hz}, 2 \mathrm{H})$, 7.59 (d, $J=8.6 \mathrm{~Hz}, 3 \mathrm{H}), 4.86(\mathrm{~s}, 2 \mathrm{H}), 2.03(\mathrm{~s}, 3 \mathrm{H}), 1.04$ (t, $J=7.0$ $\mathrm{Hz}, 9 \mathrm{H}), 0.90(\mathrm{q}, J=7.0 \mathrm{~Hz}, 6 \mathrm{H}) \mathrm{ppm} ;{ }^{13} \mathrm{C} \mathrm{NMR}\left(75 \mathrm{MHz}, \mathrm{CDCl}_{3}\right)$ $\delta=235.3,170.3,148.0,144.0,143.0,140.8,130.0,123.9,57.0$, 20.7, 7.4, 3.9 ppm; HRMS (ESI +ve) Exact mass calculated for $\mathrm{C}_{18} \mathrm{H}_{25} \mathrm{NO}_{5} \mathrm{NaSi}[\mathrm{M}+\mathrm{Na}]^{+}:$386.1394, found: 386.1392.

Ethyl 2-acetoxy-3-((triethylsilyl)carbonyl)but-3-enoate (15a): method A: yield $88 \%$ as a yellow liquid. FT-IR (film): 2956, 2877, 1746, 1606, 1371, 12118, $736 \mathrm{~cm}^{-1} ;{ }^{1} \mathrm{H}$ NMR $\left(300 \mathrm{MHz}, \mathrm{CDCl}_{3}\right) \delta$ $=6.39(\mathrm{~d}, J=0.8 \mathrm{~Hz}, 1 \mathrm{H}), 6.28(\mathrm{~s}, 1 \mathrm{H}), 5.97(\mathrm{~d}, J=0.8 \mathrm{~Hz}, 1 \mathrm{H})$, $4.28-4.04(\mathrm{~m}, 2 \mathrm{H}), 2.14(\mathrm{~s}, 3 \mathrm{H}), 1.16(\mathrm{t}, J=7.2 \mathrm{~Hz}, 3 \mathrm{H}), 0.92(\mathrm{t}$, $J=7.0 \mathrm{~Hz}, 9 \mathrm{H}, 6 \mathrm{H}), 0.77(\mathrm{q}, J=7.0 \mathrm{~Hz}, 9 \mathrm{H}) \mathrm{ppm} ;{ }^{13} \mathrm{C}$ NMR $(75$ $\left.\mathrm{MHz}, \mathrm{CDCl}_{3}\right) \delta=233.4,169.6,168.3,148.2,130.5,68.6,61.4$, 20.5, 13.8, 7.2, 3.5 ppm; HRMS (ESI+ve) Exact mass calculated for $\mathrm{C}_{15} \mathrm{H}_{26} \mathrm{O}_{5} \mathrm{NaSi}[\mathrm{M}+\mathrm{Na}]+$ : 337.1441, found: 337.1446.

2-((Dimethyl(phenyl)silyl)carbonyl)-1-phenylallyl acetate (13b): method B: yield 76\%. FT-IR (film) 2955, 2911, 2876, 1740, 1599, 1369, 1223, 10226, 735, $696 \mathrm{~cm}^{-1} ;{ }^{1} \mathrm{H}$ NMR $(500 \mathrm{MHz}$, $\left.\mathrm{CDCl}_{3}\right) \delta=7.46-7.42(\mathrm{~m}, 2 \mathrm{H}), 7.41-7.32(\mathrm{~m}, 3 \mathrm{H}), 7.31-7.23(\mathrm{~m}$, $5 \mathrm{H}), 6.60(\mathrm{~s}, 1 \mathrm{H}), 6.10(\mathrm{~s}, 1 \mathrm{H}), 6.02(\mathrm{~s}, 1 \mathrm{H}), 2.04(\mathrm{~s}, 3 \mathrm{H}), 0.51(\mathrm{~s}$, $3 \mathrm{H}), 0.45(\mathrm{~s}, 3 \mathrm{H}) \mathrm{ppm} ;{ }^{13} \mathrm{C} \mathrm{NMR}\left(126 \mathrm{MHz}, \mathrm{CDCl}_{3}\right) \delta=233.5$, $169.3,151.8,138.2,135.4,133.8,129.8,128.4,128.3,128.2$, $128.1,127.5,71.9,21.1,-3.0,-3.2$ ppm; HRMS (ESI +ve) Exact mass calculated for $\mathrm{C}_{20} \mathrm{H}_{22} \mathrm{O}_{3} \mathrm{NaSi}[\mathrm{M}+\mathrm{Na}]^{+}: 361.1236$, found: 361.1238 .

\section{2-((Dimethyl(phenyl)silyl)carbonyl)-1-(4-nitrophenyl)allyl} acetate (14b): method B: yield $80 \%$ as a yellow liquid. FT-IR (film) 2919, 2850, 1745, 1600, 1521, 1371, 1346, 1223, 1110, 1039, $834,787,734,700 \mathrm{~cm}^{-1} ;{ }^{1} \mathrm{H} \mathrm{NMR}\left(300 \mathrm{MHz}, \mathrm{CDCl}_{3}\right) \delta=8.09-8.02$ (m, 2H), 7.42-7.25 (m, 7H), $6.60(\mathrm{~s}, 1 \mathrm{H}), 6.13(\mathrm{~d}, J=1.3 \mathrm{~Hz}, 1 \mathrm{H})$, $6.04(\mathrm{~s}, 1 \mathrm{H}), 2.10(\mathrm{~s}, 3 \mathrm{H}), 0.46(\mathrm{~s}, 3 \mathrm{H}), 0.39(\mathrm{~s}, 3 \mathrm{H}) \mathrm{ppm} ;{ }^{13} \mathrm{C}$ NMR $\left(75 \mathrm{MHz}, \mathrm{CDCl}_{3}\right) \delta=233.3,169.1,150.7,147.5,145.6,134.9$, 133.7, 130.0, 129.3, 128.3, 128.2, 123.6, 71.0, 20.9, -3.1, -3.3 ppm; HRMS (ESI +ve) Exact mass calculated for $\mathrm{C}_{20} \mathrm{H}_{21} \mathrm{NO}_{5} \mathrm{NaSi}$ $[\mathrm{M}+\mathrm{Na}]^{+}:$406.1087, found: 406.1071.

\section{Ethyl-2-acetoxy-3-((dimethyl(phenyl)silyl)carbonyl)but-3-} enoate (15b): method B: yield $67 \%$ as a yellow liquid.FT-IR (film) 2924, 2853, 1745, 1608, 1372, 1217, 1115, 1060, 827, 788, 701 $\mathrm{cm}^{-1} ;{ }^{1} \mathrm{H}$ NMR $\left(400 \mathrm{MHz}, \mathrm{CDCl}_{3}\right) \delta=7.46-7.41(\mathrm{~m}, 2 \mathrm{H}), 7.33-7.25$ $(\mathrm{m}, 3 \mathrm{H}), 6.14(\mathrm{~d}, J=0.8 \mathrm{~Hz}, 1 \mathrm{H}), 6.03(\mathrm{~s}, 1 \mathrm{H}), 5.85(\mathrm{~d}, J=0.8 \mathrm{~Hz}$, $1 \mathrm{H}), 4.06$ (q, $J=7.0,2 \mathrm{H}), 2.01(\mathrm{~s}, 3 \mathrm{H}), 1.11(\mathrm{t}, J=7.1 \mathrm{~Hz}, 3 \mathrm{H})$, $0.47(\mathrm{~s}, 3 \mathrm{H}), 0,46(\mathrm{~s}, 3 \mathrm{H}) \mathrm{ppm} ;{ }^{13} \mathrm{C}$ NMR $\left(101 \mathrm{MHz}, \mathrm{CDCl}_{3}\right) \delta=$ 231.7, 169.7, 168.3, 146.7, 135.1, 133.8, 132.2, 130.0, 128.3, 68.6, 61.6, 20.6, 13.9, -3.1, -3.2 ppm; HRMS (ESI +ve) Exact mass calculated for $\mathrm{C}_{17} \mathrm{H}_{22} \mathrm{O}_{5} \mathrm{NaSi}[\mathrm{M}+\mathrm{Na}]^{+}: 357.1134$, found: 357.1134.

\section{1-(4-Nitrophenyl)-2-((trimethylsilyl)carbonyl)allylacetate}

(14c): method A: yield $72 \%$ as a yellow liquid. FT-IR (film) 1741 , 1598, 1519, 1370, 1345, 1221, 1108, 992, 839, 750, 698, 622, $530 \mathrm{~cm}^{-1} ;{ }^{1} \mathrm{HNMR}\left(300 \mathrm{MHz}, \mathrm{CDCl}_{3}\right) \delta=8.16(\mathrm{~d}, J=8.7 \mathrm{~Hz}, 2 \mathrm{H})$, $7.53(\mathrm{~d}, J=8.7 \mathrm{~Hz}, 2 \mathrm{H}, 2 \mathrm{H}), 6.67(\mathrm{~s}, 1 \mathrm{H}), 6.36(\mathrm{~d}, J=1.3 \mathrm{~Hz}, 1 \mathrm{H})$, $6.28(\mathrm{~d}, J=0.7 \mathrm{~Hz}, 1 \mathrm{H}), 2.12(\mathrm{~s}, 3 \mathrm{H}), 0.25(\mathrm{~s}, 9 \mathrm{H}) \mathrm{ppm} ;{ }^{13} \mathrm{C}$ NMR $\left(75 \mathrm{MHz}, \mathrm{CDCl}_{3}\right) \delta=235.1,169.1,150.9,147.5,145.8,128.5$, 123.6, 70.9, 20.9, -1.5 ppm; HRMS (ESI +ve) Exact mass calculated for $\mathrm{C}_{15} \mathrm{H}_{19} \mathrm{NO}_{5} \mathrm{NaSi}[\mathrm{M}+\mathrm{Na}]^{+}:$344.0924, found: 344.0928 .

\section{Ethyl-2-acetoxy-3-((trimethylsilyl)carbonyl)but-3-enoate}

(15c): method A: yield $67 \%$, method C: yield: $75 \%$ as a yellow liquid. FT-IR (film) 1742, 1608, 1371, 842, $620 \mathrm{~cm}^{-1} ;{ }^{1} \mathrm{H}$ NMR (300 $\left.\mathrm{MHz}, \mathrm{CDCl}_{3}\right) \delta=6.38(\mathrm{~s}, 1 \mathrm{H}), 6.30(\mathrm{~s}, 1 \mathrm{H}), 5.97(\mathrm{~s}, 1 \mathrm{H}), 4.21(\mathrm{q}$, $J=7.6 \mathrm{~Hz}, 2 \mathrm{H}), 2.15(\mathrm{~s}, 3 \mathrm{H}), 1.25$ (t, $J=7.6 \mathrm{~Hz}, 3 \mathrm{H}), 0.33(\mathrm{~s}, 9 \mathrm{H})$ ppm; ${ }^{13} \mathrm{C}$ NMR $\left(75 \mathrm{MHz}, \mathrm{CDCl}_{3}\right) \delta=233.4,169.7,168.4,146.9$, 130.9, 68.6, 61.6, 20.6, 13.9, -1.5 ppm; HRMS (ESI +ve) Exact mass calculated for $\mathrm{C}_{12} \mathrm{H}_{20} \mathrm{O}_{5} \mathrm{NaSi}[\mathrm{M}+\mathrm{Na}]^{+}:$295.0972, found: 295.0971 .

1-(4-Nitrophenyl)-2-((triisopropylsilyl)carbonyl)allyl acetate (14d): method B: yield $51 \%$ as a yellow liquid. FT-IR (film) 2945, 2867, 1748, 1600, 1524, 1347, 1226, 1113, 1048, 849, 751, 684 $\mathrm{cm}^{-1} ;{ }^{1} \mathrm{H}$ NMR $\left(300 \mathrm{MHz}, \mathrm{CDCl}_{3}\right) \delta=8.02(\mathrm{~d}, J=8.8 \mathrm{~Hz}, 2 \mathrm{H}), 7.37$ $(\mathrm{d}, J=8.8 \mathrm{~Hz}, 2 \mathrm{H}), 6.66(\mathrm{~s}, 1 \mathrm{H}), 6.24(\mathrm{~s}, 1 \mathrm{H}), 6.11(\mathrm{~s}, 1 \mathrm{H}), 2.05$ (s, 3H), 1.31-1.20 (m, 3H), $0.97(\mathrm{~d}, J=7.4 \mathrm{~Hz}, 9 \mathrm{H}), 0.94(\mathrm{~d}, J=$ $7.4 \mathrm{~Hz}, 9 \mathrm{H}) \mathrm{ppm} ;{ }^{13} \mathrm{C}$ NMR $\left(75 \mathrm{MHz}, \mathrm{CDCl}_{3}\right) \delta=234.9,169.1$, 153.2, 147.4, 145.9, 128.3, 127.6, 123.6, 71.3, 21.0, 18.6, 12.2 ppm; HRMS: (ESI +ve) Exact mass calculated for $\mathrm{C}_{21} \mathrm{H}_{31} \mathrm{NO}_{5} \mathrm{NaSi}$ $[\mathrm{M}+\mathrm{Na}]^{+}:$428.1869, found: 428.1869 .

Ethyl-2-acetoxy-3-((triisopropylsilyl)carbonyl)but-3-enoate (15d): method B: yield $70 \%$ as a yellow liquid. FT-IR (film) 2944 , 2867, 1748, 1602, 1464, 1371, 1216, 1062, 982, 882, $676 \mathrm{~cm}^{-1}$; ${ }^{1} \mathrm{H}$ NMR $\left(400 \mathrm{MHz}, \mathrm{CDCl}_{3}\right) \delta=6.31(\mathrm{~d}, J=0.8 \mathrm{~Hz}, 1 \mathrm{H}), 6.15(\mathrm{~s}$, $1 \mathrm{H}), 5.89(\mathrm{~s}, 1 \mathrm{H}), 4.10(\mathrm{q}, J=7.1 \mathrm{~Hz}, 2 \mathrm{H}), 2.05(\mathrm{~s}, 3 \mathrm{H}), 1.30(\mathrm{~m}$, $3 \mathrm{H}), 1.14$ (t, $J=7.1 \mathrm{~Hz}, 3 \mathrm{H}), 1.04(\mathrm{~d}, J=7.5 \mathrm{~Hz}, 9 \mathrm{H}), 1.03$ (d, $J=$ $7.5 \mathrm{~Hz}, 9 \mathrm{H}) \mathrm{ppm} ;{ }^{13} \mathrm{C} \mathrm{NMR}\left(101 \mathrm{MHz}, \mathrm{CDCl}_{3}\right) \delta=233.0$, 169.7, 
168.4, 148.9, 130.6, 69.0, 61.5, 20.6, 18.6, 18.6, 13.9, 12.2 ppm; HRMS (ESI +ve) Exact mass calculated for $\mathrm{C}_{18} \mathrm{H}_{32} \mathrm{O}_{5} \mathrm{NaSi}$ $[\mathrm{M}+\mathrm{Na}]^{+}:$379.1917, found: 379.1915 .

\section{2-((Tert-butyldimethylsilyl)carbonyl)-1-(4-nitrophenyl)allyl} acetate (14e): method C: yield $68 \%$ as a yellow liquid. FT-IR (film) 2930, 2858, 1746, 1522, 1346, 1224, 1041, 836, $780 \mathrm{~cm}^{-1} ;{ }^{1} \mathrm{H}$ $\operatorname{NMR}\left(300 \mathrm{MHz}, \mathrm{CDCl}_{3}\right) \delta=8.16(\mathrm{~d}, J=8.8 \mathrm{~Hz}, 2 \mathrm{H}), 7.53(\mathrm{~d}, J=$ $8.8 \mathrm{~Hz}, 2 \mathrm{H}), 6.69(\mathrm{~d}, J=1.1 \mathrm{~Hz}, 1 \mathrm{H}), 6.32(\mathrm{~d}, J=1.3 \mathrm{~Hz}, 1 \mathrm{H}), 6.25$ (d, $J=0.7 \mathrm{~Hz}$, ), $2.12(\mathrm{~s}, 3 \mathrm{H}), 0.84(\mathrm{~s}, 9 \mathrm{H}), 0.25$ (s, 3H), $0.24(\mathrm{~s}$, $3 \mathrm{H}) \mathrm{ppm} ;{ }^{13} \mathrm{C}$ NMR $\left(75 \mathrm{MHz}, \mathrm{CDCl}_{3}\right) \delta=234.7,217.1,210.3$, 169.1, 152.6, 147.4, 145.8, 128.6, 128.2, 123.5, 71.1, 26.5, 20.9, 16.7, -4.8, -4.9 ppm; HRMS (ESI +ve) Exact mass calculated for $\mathrm{C}_{18} \mathrm{H}_{25} \mathrm{NO}_{5} \mathrm{NaSi}[\mathrm{M}+\mathrm{Na}]+:$ 386.1394, found: 386.1397.

Ethyl-2-acetoxy-3-((tert-butyldimethylsilyl)carbonyl)but-3enoate (15e): method C: yield $84 \%$ as a yellow liquid. FT-IR (film) 2928, 2857, 1749, 1602, 1465, 1370, 1248, 1220, 839, $778 \mathrm{~cm}^{-1}$; ${ }^{1} \mathrm{H}$ NMR $\left(300 \mathrm{MHz}, \mathrm{CDCl}_{3}\right) \delta=6.35(\mathrm{~d}, J=0.7 \mathrm{~Hz}, 1 \mathrm{H}), 6.27(\mathrm{~s}$, $1 \mathrm{H}), 5.94(\mathrm{~d}, J=0.7 \mathrm{~Hz}, 1 \mathrm{H}), 4.17(\mathrm{q}, J=7.1 \mathrm{~Hz}, 2 \mathrm{H}), 2.11(\mathrm{~s}, 3 \mathrm{H})$, $1.22(\mathrm{t}, J=7.1 \mathrm{~Hz}, 3 \mathrm{H}), 0.92(\mathrm{~s}, 9 \mathrm{H}), 0.28(\mathrm{~d}, J=2.9 \mathrm{~Hz}, 3 \mathrm{H}), 0.27$ ppm; ${ }^{13} \mathrm{C}$ NMR $\left(75 \mathrm{MHz}, \mathrm{CDCl}_{3}\right) \delta=233.0,169.6,168.4,148.4$, $131.4,68.9,61.5,26.5,20.6,16.8,13.9,-5.0$ ppm; HRMS (ESI + ve) Exact mass calculated for $\mathrm{C}_{15} \mathrm{H}_{26} \mathrm{O}_{5} \mathrm{NaSi}[\mathrm{M}+\mathrm{Na}]+: 337.1441$ found: 337.1440 .

\section{2-((Methyldiphenylsilyl)carbonyl)-1-(4-nitrophenyl)allyl} acetate (14f): method A: yield: $79 \%$, method C: yield $54 \%$ as a yellow liquid. FT-IR (film) 2953, 2923, 2878, 17448, 1599, 1522, $1343,1218,851,742,684 \mathrm{~cm}^{-1} ;{ }^{1} \mathrm{H}$ NMR $\left(300 \mathrm{MHz}, \mathrm{CDCl}_{3}\right) \delta=$ $8.34-7.90(\mathrm{~m}, 2 \mathrm{H}), 7.71-7.14(\mathrm{~m}, 10 \mathrm{H}), 6.78(\mathrm{~s}, 1 \mathrm{H}), 6.27(\mathrm{~d}, J$ $=1.4 \mathrm{~Hz}, 1 \mathrm{H}), 6.19(\mathrm{~s}, 1 \mathrm{H}), 2.12(\mathrm{~s}, 3 \mathrm{H}), 0.78(\mathrm{~s}, 3 \mathrm{H}) \mathrm{ppm} ;{ }^{13} \mathrm{C}$ NMR $\left(75 \mathrm{MHz}, \mathrm{CDCl}_{3}\right): \delta=231.7,169.1,151.3,147.5,145.6$, $134.9,134.9$ (2C), 133.1, 132.9, 130.3, 130.2, 128.3(3C), 123.6, 71.1, 21.0, -3.6 ppm; HRMS (ESI +ve) Exact mass calculated for $\mathrm{C}_{25} \mathrm{H}_{23} \mathrm{NO}_{5} \mathrm{NaSi}[\mathrm{M}+\mathrm{Na}]^{+}:$468.1237, found: 468.1237.

\section{Ethyl-2-acetoxy-3-((methyldiphenylsilyl)carbonyl)but-3-} enoate (15f): method C: yield $77 \%$ as a yellow liquid. FT-IR (film) 2959, 2925, 2853, 1746, 1020, 800, 722, $700 \mathrm{~cm}^{-1} ;{ }^{1} \mathrm{H}$ NMR $(300$ $\left.\mathrm{MHz}, \mathrm{CDCl}_{3}\right) \delta=7.67-7.56(\mathrm{~m}, 4 \mathrm{H}), 7.51-7.29(\mathrm{~m}, 6 \mathrm{H}), 6.29$ (s, 1H), $6.19(\mathrm{~s}, 1 \mathrm{H}), 6.03(\mathrm{~s}, 1 \mathrm{H}), 4.18(\mathrm{q}, J=7.1 \mathrm{~Hz}, 2 \mathrm{H}), 2.14$ (s, 3H), 1.21 (t, $J=7.1 \mathrm{~Hz}, 3 \mathrm{H}), 0.83(\mathrm{~s}, 3 \mathrm{H}) \mathrm{ppm} ;{ }^{13} \mathrm{C}$ NMR $(75$ $\left.\mathrm{MHz}, \mathrm{CDCl}_{3}\right) \delta=230.1,169.7,168.3,147.4,135.0,133.3,133.2$, $133.1,130.3,128.3,128.3,68.7,61.7,20.7,14.0,-3.5$ ppm; HRMS (ESI +ve) Exact mass calculated for $\mathrm{C}_{22} \mathrm{H}_{24} \mathrm{O}_{5} \mathrm{NaSi}$ $[\mathrm{M}+\mathrm{Na}]^{+}:$419.12852, found: 419.1282 .

\section{(E)-3-(4-Nitrophenyl)-2-((triethylsilyl)carbonyl)allylacetate}

(14'a): in a $10 \mathrm{~mL}$ round bottomed was added compound (14a) $(610 \mathrm{mg}, 1.67 \mathrm{mmol})$ and $1.5 \mathrm{ml}$ of $\left[\mathrm{BMiM}, \mathrm{PF}_{6}\right.$ followed by addition of KOAc (214 mg, $2.2 \mathrm{mmol}, 1.5$ equiv) at $50{ }^{\circ} \mathrm{C}$. The reaction mixture was kept for 2 hours at this temperature. The product was extracted with diethyl ether and purified by column chromatography with $20 \%$ diethyl ether/cyclohexane as eluent to afford pure product (14'a) (386 mg, 64\% yield) as a yellow solid. FT-IR (Film) 2956, 2876, 1738, 1597, 1522, 1339, 1222, 1097, $1020,856,724,702 \mathrm{~cm}^{-1} ;{ }^{1} \mathrm{H}$ NMR $\left(300 \mathrm{MHz}, \mathrm{CDCl}_{3}\right) \delta=8.30(\mathrm{~d}$,
$J=8.8 \mathrm{~Hz}, 2 \mathrm{H}), 7.59(\mathrm{~d}, J=8.8 \mathrm{~Hz}, 2 \mathrm{H}), 7.57(\mathrm{~s}, 1 \mathrm{H}), 4.86(\mathrm{~s}, 2 \mathrm{H})$, 2.03 (s, 3H), 1.04 (t, J=6.7 Hz, 9H), 0.90 (q, J=6.7 Hz, 6H) ppm; ${ }^{13} \mathrm{C}$ NMR $\left(75 \mathrm{MHz}, \mathrm{CDCl}_{3}\right) \delta=235.3,170.3,148.0,144.0,143.0$, 140.8, 130.0, 123.9, 57.0, 20.7, 7.4, 3.9 ppm; HRMS (ESI +ve) Exact mass calculated for $\mathrm{C}_{18} \mathrm{H}_{25} \mathrm{NO}_{5} \mathrm{NaSi}[\mathrm{M}+\mathrm{Na}]+: 386.13942$ found: 386.1392 .

(E)-3-(4-nitrophenyl)-2-((trimethylsilyl)carbonyl)allylacetate (14'c): yield $72 \%$ as a yellow thick oil. FT-IR (Film) 2960, 1738, $1597,1517,1350,1229,1028,838 \mathrm{~cm}^{-1} ;{ }^{1} \mathrm{H}$ NMR $(300 \mathrm{MHz}$, $\left.\mathrm{CDCl}_{3}\right) \delta=8.25(\mathrm{~d}, \mathrm{~J}=8.8 \mathrm{~Hz}, 2 \mathrm{H}), 7.60(\mathrm{~d}, J=8.8 \mathrm{~Hz}, 2 \mathrm{H}), 7.56$ (s, 1H), 4.81(s, 2H), $2.03(\mathrm{~s}, 3 \mathrm{H}), 0.31(\mathrm{~s}, 9 \mathrm{H}) \mathrm{ppm} ;{ }^{13} \mathrm{C}$ NMR $(75$ $\left.\mathrm{MHz}, \mathrm{CDCl}_{3}\right) \delta=235.0,170.3,147.9,143.7,142.6,140.7,130.1$, 123.8, 57.0, 20.7, -1.2 ppm; HRMS (ESI +ve) Exact mass calculated for $\mathrm{C}_{15} \mathrm{H}_{19} \mathrm{NO}_{5} \mathrm{NaSi}[\mathrm{M}+\mathrm{Na}]+$ : 344.1924, found: 344.0925 .

\section{(E)-2-((tert-butyldimethylsilyl)carbonyl)but-2-en-1-ylacetate} (14'e): yield $81 \%$ as a yellow thick oil. FT-IR (Film) 2960, 2931, 2855, 1739, 1512, 1345, 1227, 735 $\mathrm{cm}^{-1}$; ${ }^{1} \mathrm{H}$ NMR $(300 \mathrm{MHz}$, $\left.\mathrm{CDCl}_{3}\right) \delta=8.29(\mathrm{~d}, J=8.8 \mathrm{~Hz}, 2 \mathrm{H}), 7.59(\mathrm{~d}, \mathrm{~J}=8.8 \mathrm{~Hz}, 2 \mathrm{H}), 7.56$ (d, $J=2.1 \mathrm{~Hz}, 1 \mathrm{H}$ ), $4.85(\mathrm{~s}, 2 \mathrm{H}), 2.04(\mathrm{~s}, 3 \mathrm{H}), 1.00(\mathrm{~s}, 9 \mathrm{H}), 0.38$ (s, 6H) ppm; ${ }^{13} \mathrm{C}$ NMR $\left(75 \mathrm{MHz}, \mathrm{CDCl}_{3}\right) \delta=234.9,170.4,148.0$, 144.2, 143.7, 140.8, 129.9, 123.9, 57.2, 26.7, 20.8, 17.0, -4.5 ppm; HRMS (ESI +ve) Exact mass calculated for $\mathrm{C}_{18} \mathrm{H}_{25} \mathrm{NO}_{5} \mathrm{NaSi}$ $[\mathrm{M}+\mathrm{Na}]+:$ 386.1394, found: 386.1392 .

Ethyl-(E)-4-acetoxy-3-((tert-butyldimethylsilyl)carbonyl)pent2-enoate (15'e): yield $64 \%$ as a yellow thick oil. FT-IR (Film) 2945 , 2932, 2859, 1751, 1226, 1202, 1032, 838, $779 \mathrm{~cm}^{-1} ;{ }^{1} \mathrm{H}$ NMR $(300$ $\left.\mathrm{MHz}, \mathrm{CDCl}_{3}\right) \delta=6.35(\mathrm{~s}, 1 \mathrm{H}), 5.19(\mathrm{~s}, 2 \mathrm{H}), 4.27(\mathrm{q}, J=7.1 \mathrm{~Hz}$, 2H), $2.01(\mathrm{~s}, 3 \mathrm{H}), 1.34(\mathrm{t}, J=7.1 \mathrm{~Hz}, 3 \mathrm{H}), 0.96(\mathrm{~s}, 9 \mathrm{H}), 0.31(\mathrm{~s}$, $6 \mathrm{H}) \mathrm{ppm} ;{ }^{13} \mathrm{C}$ NMR $\left(75 \mathrm{MHz}, \mathrm{CDCl}_{3}\right) \delta=237.5,170.2,165.3$, 153.1, 128.2, 61.3, 58.0, 26.5, 20.7, 17.0, 14.1, -5.3 ppm; HRMS $(\mathrm{ESI}+\mathrm{ve})$ Exact mass calculated for $\mathrm{C}_{15} \mathrm{H}_{26} \mathrm{O}_{5} \mathrm{NaSi}[\mathrm{M}+\mathrm{Na}]+$ : 337.14417, found: 337.1438 .

General experimental procedure for the transformation of MBH adducts into aldehydes:

A round bottom flask was charged with an acetyl protected $\beta$ hydroxyacryloylsilane dissolved in acetone/water $2: 1$ or THF/water 9:1. The solution was degasified then enlightened at $365 \mathrm{~nm}$ under stirring at room temperature. After completion of the reaction as monitored by TLC, solvents were removed in vacuum. The residue was dissolved in DCM and an aqueous workup done. The organic layer was dried with $\mathrm{MgSO}_{4}$ and after concentration under reduced pressure the corresponding aldehyde was obtained.

2-Formyl-1-phenylallyl acetate (16): from compound (13a): yield $95 \%$; from compound (13b): yield $88 \%$ as colourless oil. FTIR (film) 2960, 1737, 1688, 1222, 1025, $697 \mathrm{~cm}^{-1} ;{ }^{1} \mathrm{H}$ NMR $(500$ $\left.\mathrm{MHz}, \mathrm{CDCl}_{3}\right) \delta=9.56(\mathrm{~s}, 1 \mathrm{H}), 7.44-7.27(\mathrm{~m}, 5 \mathrm{H}), 6.68(\mathrm{~s}, 1 \mathrm{H})$, $6.51(\mathrm{~d}, J=1.4 \mathrm{~Hz}, 1 \mathrm{H}), 6.22(\mathrm{~s}, 1 \mathrm{H}), 2.13(\mathrm{~s}, 3 \mathrm{H}) \mathrm{ppm} ;{ }^{13} \mathrm{C}$ NMR $\left(125 \mathrm{MHz}, \mathrm{CDCl}_{3}\right) \delta=191.7,169.3,148.7,137.4,133.6,128.5$, 128.4, 127.4, 71.4, 21.0 ppm; HRMS (ESI +ve) Exact mass calculated for $\mathrm{C}_{12} \mathrm{H}_{12} \mathrm{O}_{3} \mathrm{Na}[\mathrm{M}+\mathrm{Na}]^{+}:$227.0678, found: 227.0681 . 
2-Formyl-1-(4-nitrophenyl)allyl acetate (17): from compound (14a): yield 92\%; (14b): yield 88\%; (14d): yield 75\%; (14e): yield 91\%; (14f): yield $69 \%$ as a colourless oil. FT-IR (film) 3088,2935 , 2858, 1741, 1687, 1602, 1518, 1335, 1225, 967, 848, 747, 695 $\mathrm{cm}^{-1} ;{ }^{1} \mathrm{H}$ NMR $\left(300 \mathrm{MHz}, \mathrm{CDCl}_{3}\right) \delta=9.54(\mathrm{~s}, 1 \mathrm{H}), 8.17(\mathrm{~d}, J=8.8$ $\mathrm{Hz}, 2 \mathrm{H}), 7.56(\mathrm{~d}, J=8.8 \mathrm{~Hz}, 2 \mathrm{H}), 6.69(\mathrm{~s}, 1 \mathrm{H}), 6.60(\mathrm{~d}, J=1.3 \mathrm{~Hz}$, $1 \mathrm{H}), 6.29$ (s, 1H), 2.15 (s, 3H) ppm; ${ }^{13} \mathrm{C}$ NMR $\left(75 \mathrm{MHz}, \mathrm{CDCl}_{3}\right) \delta$ = 191.4, 169.1, 147.7, 144.7, 134.5, 128.2, 123.7, 70.4, 20.8 ppm; HRMS (ESI +ve) Exact mass calculated for $\mathrm{C}_{12} \mathrm{H}_{11} \mathrm{NO}_{5} \mathrm{Na}$ $[\mathrm{M}+\mathrm{Na}]+:$ 272.05294, found: 272.0528 .

Ethyl 2-acetoxy-3-formylbut-3-enoate (18): from compound (15a): yield 53\%; (15b): yield 83\%; (15c): yield 95\%; (15d): yield $80 \%$; (15e): yield $95 \%$; (15f): yield $81 \%$ as a colourless oil. FTIR (film) 2924, 2852, 1723, 1450, 1370, 1210, 1158, 1030, 883 $\mathrm{cm}^{-1} ;{ }^{1} \mathrm{H}$ NMR $\left(400 \mathrm{MHz}, \mathrm{CDCl}_{3}\right) \delta=9.52(\mathrm{~s}, 1 \mathrm{H}), 6.55(\mathrm{~s}, 1 \mathrm{H})$, $6.29(\mathrm{~s}, 1 \mathrm{H}), 5.89(\mathrm{~d}, J=1.0 \mathrm{~Hz}, 1 \mathrm{H}), 4.15(\mathrm{q}, J=7.2 \mathrm{~Hz}, 2 \mathrm{H})$, 2.09 (s, 3H), $1.20-1.16(\mathrm{t}, J=7.2 \mathrm{~Hz}, 3 \mathrm{H}) \mathrm{ppm} ;{ }^{13} \mathrm{C}$ NMR $(101$ $\left.\mathrm{MHz}, \mathrm{CDCl}_{3}\right) \delta=190.6,169.6,167.6,143.5,137.3,67.5,62.0$, 20.6, 13.9 ppm; HRMS (ESI +ve) Exact mass calculated for $\mathrm{C}_{9} \mathrm{H}_{12} \mathrm{O}_{5} \mathrm{Na}[\mathrm{M}+\mathrm{Na}]^{+}:$223.0582, found: 223.0584 .

2-(Hydroxy(phenyl)methyl)acrylaldehyde (19): from compound (4a): yield $61 \%$ as a colourless oil. FT-IR (film) 3412 , 2922, 1682, 1453, 958, $698 \mathrm{~cm}^{-1} ;{ }^{1} \mathrm{H}$ NMR $\left(400 \mathrm{MHz}, \mathrm{CDCl}_{3}\right) \delta=$ $9.59(\mathrm{~s}, 1 \mathrm{H}), 7.41-7.27(\mathrm{~m}, 5 \mathrm{H}), 6.47(\mathrm{~d}, J=1.4 \mathrm{~Hz}, 1 \mathrm{H}), 6.18(\mathrm{~s}$, $1 \mathrm{H}), 5.64(\mathrm{~s}, 1 \mathrm{H}), 2.88(\mathrm{~d}, J=3.5 \mathrm{~Hz}, 1 \mathrm{H}) \mathrm{ppm} ;{ }^{13} \mathrm{C}$ NMR $(101$ $\left.\mathrm{MHz}, \mathrm{CDCl}_{3}\right) \delta=193.9,151.3,140.8,134.5,128.5,128.0,126.6$, $71.1 \mathrm{ppm}$; HRMS (ESI +ve) Exact mass calculated for $\mathrm{C}_{10} \mathrm{H}_{10} \mathrm{O}_{2} \mathrm{Na}[\mathrm{M}+\mathrm{Na}]^{+}:$185.0573, found: 185.0574 .

2-(Hydroxy(4-nitrophenyl)methyl) acrylaldehyde (20): from compound (6a): yield 78\%; (6c): yield $86 \%$; $(6 \mathrm{e})$ : yield $86 \%$; $(6 \mathrm{f})$ : yield $93 \%$ as a colourless oil. FT-IR (film) $3071,2958,2855,1688$, $1600,1515,1428,1343,1255,1111,850,691 \mathrm{~cm}^{-1} ;{ }^{1} \mathrm{H}$ NMR $(300$ $\left.\mathrm{MHz}, \mathrm{CDCl}_{3}\right) \delta=9.56(\mathrm{~s}, 1 \mathrm{H}), 8.16(\mathrm{~d}, J=8.8 \mathrm{~Hz}, 2 \mathrm{H}), 7.56(\mathrm{~d}, J$ $=8.8 \mathrm{~Hz}, 2 \mathrm{H}), 6.52(\mathrm{~d}, J=1.3 \mathrm{~Hz}, 1 \mathrm{H}), 6.23(\mathrm{~d}, J=0.7 \mathrm{~Hz}, 1 \mathrm{H})$, $5.71(\mathrm{~s}, 1 \mathrm{H}) \mathrm{ppm} ;{ }^{13} \mathrm{C} \mathrm{NMR}\left(75 \mathrm{MHz}, \mathrm{CDCl}_{3}\right) \delta=193.6,150.5$, 148.4, 147.4, 135.3, 127.4, 123.6, 69.9 ppm; HRMS (ESI +ve) Exact mass calculated for $\mathrm{C}_{10} \mathrm{H}_{8} \mathrm{NO}_{3}\left[\mathrm{M}-\mathrm{H}_{2} \mathrm{O}+\mathrm{H}\right]^{+}:$190.0498, found: 191.0498 .

Ethyl 3-formyl-2-hydroxybut-3-enoate (21): from compound (11a): yield 81\%; (11c): yield 53\%; (11e): yield 63\%; (11f): yield $74 \%$ as a colourless oil. FT-IR (film) 3458, 2982, 2936, 1734, 1687, 1446, 1200, 1022, $969 \mathrm{~cm}^{-1}$; ${ }^{1} \mathrm{H}$ NMR $\left(300 \mathrm{MHz}, \mathrm{CDCl}_{3}\right) \delta$ $9.60(\mathrm{~s}, 1 \mathrm{H}), 6.58(\mathrm{~d}, J=0.9 \mathrm{~Hz}, 1 \mathrm{H}), 6.27(\mathrm{~s}, 1 \mathrm{H}), 4.94(\mathrm{~s}, 1 \mathrm{H})$, 4.25 (q, $J=7.2 \mathrm{~Hz}, 2 \mathrm{H}$ ), 1.26 (t, $J=7.2 \mathrm{~Hz}, 3 \mathrm{H}$ ) ppm; ${ }^{13} \mathrm{C} \mathrm{NMR}$ $\left(75 \mathrm{MHz}, \mathrm{CDCl}_{3}\right.$ ) $\delta 192.1,172.2,146.7,136.3,68.2,62.4,13.9$ ppm; HRMS: (ESI +ve) Exact mass calculated for $\mathrm{C}_{7} \mathrm{H}_{10} \mathrm{O}_{4} \mathrm{Na}$ $[\mathrm{M}+\mathrm{Na}]+:$ 181.04713, found: 181.0474 .

2-Methylene-5-oxo-5-(triethylsilyl)pentanal (22): from compound 5a: yield $60 \%$ as a colourless oil. FT-IR (film) 2954, 2876, 1689, 1639, 1414, 1016, 945, $719 \mathrm{~cm}^{-1} ;{ }^{1} \mathrm{H}$ NMR $(500 \mathrm{MHz}$, $\left.\mathrm{CDCl}_{3}\right) \delta=9.51(\mathrm{~s}, 1 \mathrm{H}), 6.26(\mathrm{~s}, 1 \mathrm{H}), 5.99(\mathrm{~s}, 1 \mathrm{H}), 2.75(\mathrm{t}, J=7.2$ $\mathrm{Hz}, 2 \mathrm{H}$ ), 2.48 (t, $J=7.2 \mathrm{~Hz}, 2 \mathrm{H}$ ), 0.96 (t, $J=8.0 \mathrm{~Hz}, 9 \mathrm{H}$ ), 0.73 (q, $J=8.0 \mathrm{~Hz}, 6 \mathrm{H}) \mathrm{ppm} ;{ }^{13} \mathrm{C} \mathrm{NMR}\left(126 \mathrm{MHz}, \mathrm{CDCl}_{3}\right) \delta=245.7,194.7$,
149.6, 134.9, 47.9, 20.7, 7.2, 2.0 ppm; HRMS (ESI +ve) Exact mass calculated for $\mathrm{C}_{12} \mathrm{H}_{22} \mathrm{O}_{2} \mathrm{NaSi}[\mathrm{M}+\mathrm{Na}]^{+}:$249.1281, found: 249.1281 .

5-(Dimethyl(phenyl)silyl)-2-methylene-5-oxopentanal (23): from compound $\mathbf{5 b}$ : $90 \%$ yield as a colourless oil. FT-IR (film) 2924, 2859, 1690, 1642, 1428, 1252, 1112, 950, 815, 783, 736, $649 \mathrm{~cm}^{-1} ;{ }^{1} \mathrm{H}$ NMR $\left(300 \mathrm{MHz}, \mathrm{CDCl}_{3}\right) \delta=9.43(\mathrm{~s}, 1 \mathrm{H}), 7.57-7.46$ $(\mathrm{m}, 2 \mathrm{H}), 7.46-7.29(\mathrm{~m}, 3 \mathrm{H}), 6.13(\mathrm{~s}, 1 \mathrm{H}), 5.91(\mathrm{~s}, 1 \mathrm{H}), 2.75(\mathrm{t}, J=$ $7.0 \mathrm{~Hz}, 2 \mathrm{H}$ ), 2.42 (t, $J=7.0 \mathrm{~Hz}, 2 \mathrm{H}), 0.49$ (s, 6H) ppm; ${ }^{13} \mathrm{C}$ NMR $\left(75 \mathrm{MHz}, \mathrm{CDCl}_{3}\right) \delta=244.2,194.3,149.0,134.8,133.9,133.0$, 129.9, 128.2, 46.3, 20.8, -4.9 ppm; HRMS (ESI +ve) Exact mass calculated for $\mathrm{C}_{14} \mathrm{H}_{18} \mathrm{O}_{2} \mathrm{NaSi}[\mathrm{M}+\mathrm{Na}]^{+}: 269.0974$, found: 269.0977 .

2-Methylene-5-oxo-5-(triisopropylsilyl)pentanal (24): from compound 5d: yield $90 \%$ as a colourless oil. FT-IR (film) 2942 , 2866, 1692, 1636, 1464, 1251, 881, 828, $674 \mathrm{~cm}^{-1} ;{ }^{1} \mathrm{H}$ NMR $(300$ $\left.\mathrm{MHz}, \mathrm{CDCl}_{3}\right) \delta=9.44(\mathrm{~s}, 1 \mathrm{H}), 6.22(\mathrm{~s}, 1 \mathrm{H}), 5.93(\mathrm{~s}, 1 \mathrm{H}), 2.68(\mathrm{t}, J$ $=7.1 \mathrm{~Hz}, 2 \mathrm{H}), 2.42(\mathrm{t}, J=7.1 \mathrm{~Hz}, 2 \mathrm{H}), 1.28-1.14(\mathrm{~m}, 3 \mathrm{H}), 1.02(\mathrm{~s}$, 9H), 0.98 (s, 9H) ppm; ${ }^{13} \mathrm{C} \mathrm{NMR}\left(75 \mathrm{MHz}, \mathrm{CDCl}_{3}\right) \delta=245.3,194.4$, 149.4, 134.9, 49.1, 20.6, 18.5, 17.6, 10.7 ppm; HRMS (ESI +ve) Exact mass calculated for $\mathrm{C}_{15} \mathrm{H}_{28} \mathrm{O}_{2} \mathrm{NaSi}[\mathrm{M}+\mathrm{Na}]^{+}:$291.1756, found: 291.1756 .

\section{Acknowledgments}

This research has been performed as part of the Indo-French "Joint Laboratory for Natural Products and Synthesis toward Affordable Health". We thank CNRS, CSIR and University of Rennes 1 for support of this research. G. M. thanks to CSIR for financial assistance. We thank CRMPO (Rennes) for MS analyses. We thank a reviewer for useful comments on literature dealing with $\mathrm{MBH}$ reactions on acrolein.

Keywords: Morita-Baylis-Hillman • Rauhut-Currier • Acylsilanes - Photolysis • Enals

[1] For comprehensive reviews on $\mathrm{MBH}$ reactions see: (a) V. Singh, S. Batra, Tetrahedron 2008, 64, 4511-4574; (b) D. Basavaiah, B. S. Reddy, S. S. Batsara, Chem. Rev. 2010, 110, 5447-5674; (c) D. Basavaiah, G. Veerraraghavaiah, Chem. Soc. Rev. 2012, 41, 68-78; (d) Y. Wei, M. Shi, Chem. Rev. 2013, 113, 6659-6690; (e) H. Pelissier, Tetrahedron 2017, 73, 2831-2861; (f) T. N. Reddy, V. J. Rao, Tetrahedron Lett. 2018, 59, 2859-2875 and references cited therein

[2] (a) J. S. Hill, N. S. Isaacs, Tetrahedron Lett. 1986, 27, 5007-5010; (b) G. M. Strunz, R. Bethell, G. Sampson, P. White, Can. J. Chem. 1995, 73 , 1666-1674; (c) D. Basavaiah, A. J. Rao, M. Krishnamacharyulu, Arkivoc 2002, vii, 136-145; (d) Y-M. Xu, M. Shi, J. Org. Chem. 2004, 69, 417425; (e) X. Tang, B. Zhang, Z. He, R. Gao, Z. He, Adv. Synth. Cat. 2007, 349, 2007-2017; (f) X. Zeng, Y. Liu, C. Ji, J. Zhou Chin. J. Chem. 2012, 30, 2631-2635.

[3] P. Rada Krishna, R. R. Kadiyala, Tetrahedron Lett. 2010, 51, 4981-4983.

[4] For excellent reviews on acylsilanes and $\alpha-\beta$ unsaturated acylsilanes see: (a) A. F. Patrocinio, P. J. S. Moran, J. Braz. Chem. Soc. 2001, 12, 7-31; (b) P. C. B. Page, M. J. McKenzie, Product Subclass 25: Acylsilanes. In Science of Synthesis; I. Fleming, Ed.; Thieme: Stuttgart, 2001; Vol. 4, pp 513-568; (c) M. N. Garrett, J. S. Johnson, Product Subclass 4: Silicon compound. In Science of Synthesis; I. Fleming, Ed.; Thieme: Stuttgart, Knowledge update 2012/2; Vol. 4, pp 1-85; (d) G. R. Boyce, S. N. Grezler, J. S. Johnson, X. Linghu, J. T. Malinovski, D. A. Nicewicz, A. D. Satterfield, D. C. Schmitt, K. M. Steward, J. Org. Chem. 
2012, 77, 4503-4515; (e) H.-J. Zhang, D. L. Pribbenow, C. Bolm, Chem. Soc. Rev. 2013, 42, 8540-8571 and references cited therein.

[5] J. Ruiz, A. S. Murthy, T. Roisnel, S. Chandrasekhar, R. Grée, J. Org. Chem. 2015, 80, 2364-2375.

[6] S. Denmark, M. Xie, J. Org. Chem. 2007, 72, 7050-7053.

[7] a) J. C. Depezay, Y. Le Merrer, Tetrahedron Lett. 1974, 32, 2751-2754 b) J. C. Depezay, Y. Le Merrer, Bull. Soc. Chim. Fr. 1981, 7-8 (Pt. II) 306-312.

[8] P. Singh, A. Kumar, S. Kaur, J. Kaur, H. Singh, Chem. Commun., 2016, 52, 2936-2939.

[9] L. De Luca, G. Giocomelli, A. Porcheddu, J. Org. Chem. 2001, 66, 79077909.

[10] Elegant uses of $\alpha-\beta$-unsaturated acylsilanes in synthesis have been already described, see for instance: A. Degl'Innocenti, P. Ulivi, A Capperucci, G. Reginato, A. Mordini, A. Ricci, Synlett, 1992, 883-886, and references cited therein.
[11] For a review article on Rauhut-Currier reactions see: C. E. Aroyan, A. Dermenci, S. J. Miller, Tetrahedron 2009, 65, 4069-4084.

[12] G. W. Kabalka, B. Venkataiah, G. Dong, Tetrahedron Lett. 2003, 44, 4673-4675.

[13] We used a Vilber Lourmat VL-6.LC lamp equiped with two $6 \mathrm{~W}$ bulbs at $365 \mathrm{~nm}$ for the small scale reactions, or a LT5W T8/010 UV Dudexa lamp from Narva for the larger scale reactions (see supplementary information).

[14] For instance, very few derivatives with an $\alpha$-methylene-1,3- diol structure have been reported to date. All of them are symmetrical and only a few synthetic uses have been described for them: a) C. M. König, K. Harms, U. Koert, Org. Lett. 2007, 9, 4777-4779; b) Y. Lu, I. S. Kim, A. Hassan, D. J. Del Valle, M. J. Krische, Ang. Chem. 2009, 121, 5118-5121; Ang Chem. Int. Ed. 2009, 48, 5018-5021; c) A. F. G. Goldberg, R. A. Craig II, N. R. O'Connor, B. M. Stoltz, Tetrahedron Lett. 2015, 56, 2983-2990. 


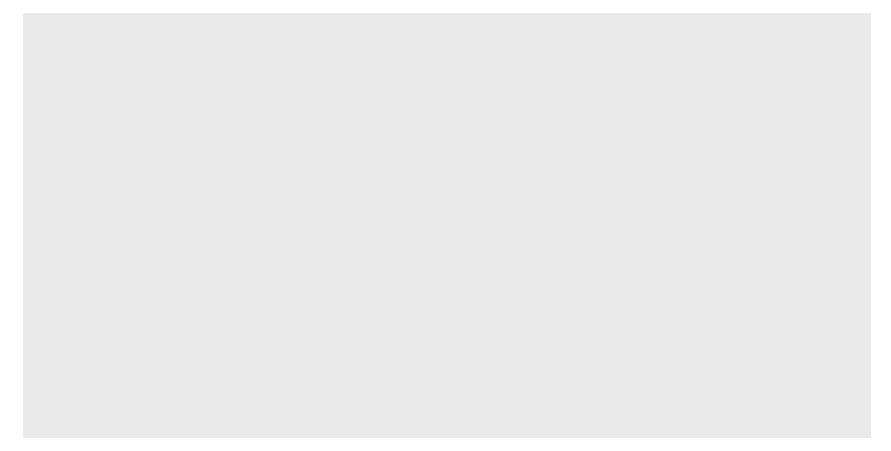

\section{Key Topic: Synthetic Methodology}

Gangababu Marri, Frédéric Justaud, Saibal Das and René Grée

$\alpha-\beta$ Unsaturated Acylsilanes as Surrogates of Acrolein for MoritaBaylis-Hillman Reactions

A short approach toward new acrolein-type derivatives, functionalized in position 2 is described. It involves as the key step, a smooth transformation of acylsilanes into aldehydes by irradiation in water-organic solvent mixtures. The functionalized enals obtained by this new route appear as versatile building blocks for the preparation of natural products and/or bioactive compounds. 